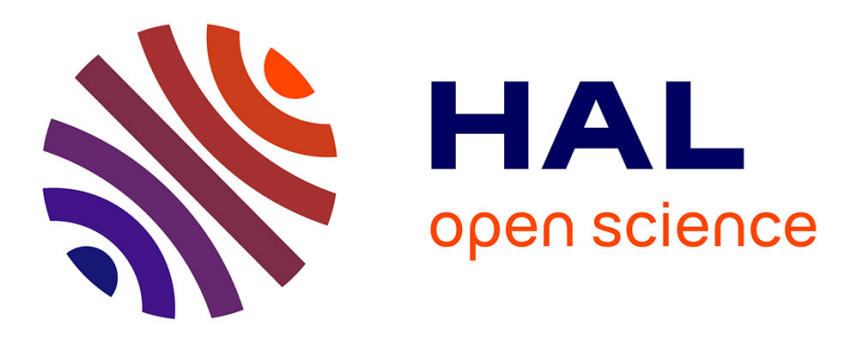

\title{
Structural Odd-Even Effect Impacting the Dimensionality of Transport in BTBT-CnOH Organic Field Effect Transistors
}

Gilles Roche, Gudrun Bruckner, Dan Dumitrescu, Joël Moreau, Arie van Der Lee, Guillaume Wantz, O.J. Dautel

\section{To cite this version:}

Gilles Roche, Gudrun Bruckner, Dan Dumitrescu, Joël Moreau, Arie van Der Lee, et al.. Structural Odd-Even Effect Impacting the Dimensionality of Transport in BTBT-CnOH Organic Field Effect Transistors. Advanced Electronic Materials, In press, Advanced Electronic Materials, pp.2100265. 10.1002/aelm.202100265 . hal-03388528

\section{HAL Id: hal-03388528 https://hal.science/hal-03388528}

Submitted on 20 Oct 2021

HAL is a multi-disciplinary open access archive for the deposit and dissemination of scientific research documents, whether they are published or not. The documents may come from teaching and research institutions in France or abroad, or from public or private research centers.
L'archive ouverte pluridisciplinaire HAL, est destinée au dépôt et à la diffusion de documents scientifiques de niveau recherche, publiés ou non, émanant des établissements d'enseignement et de recherche français ou étrangers, des laboratoires publics ou privés. 


\section{WILEY-VCH}

Structural Odd-Even Effect Impacting the Dimensionality of Transport in BTBT-CnOH Organic Field Effect Transistors

Gilles H. Roche ${ }^{1,2}$, Gudrun Bruckner ${ }^{1}$, Dan G. Dumitrescu ${ }^{3}$, Joël J. E. Moreau ${ }^{1}$, Arie van der Lee $^{4}$, Guillaume Wantz ${ }^{2}$, Olivier J. Dautel ${ }^{1}$

Gilles H. Roche

1. ICGM, Université de Montpellier, CNRS, ENSCM, Montpellier, France

2. Université de Bordeaux, IMS, CNRS, UMR-5218, Bordeaux INP, 33405 Talence, France.

Gudrun Bruckner

1. ICGM, Université de Montpellier, CNRS, ENSCM, Montpellier, France

Dan G. Dumitrescu,

3. Elettra, Sincrotrone Trieste S.C.p.A., Strada Statale 14 - km 163,5 in AREA Science Park, Basovizza, Trieste, 34149, Italy

Joël J. Moreau,

1. ICGM, Université de Montpellier, CNRS, ENSCM, Montpellier, France

Arie van der Lee,

4. IEM, Université de Montpellier, CNRS, ENSCM, Montpellier, France

Guillaume Wantz,

2. Université de Bordeaux, IMS, CNRS, UMR-5218, Bordeaux INP, 33405 Talence, France.

Olivier J. Dautel,

1. ICGM, Université de Montpellier, CNRS, ENSCM, Montpellier, France

Keywords: organic semiconductor, OFET, odd-even effect

Abstract. We report the synthesis and characterization of a series of [1]benzothieno[3,2-

b]benzothiophene (BTBT) molecules disubstituted by hydroxy aliphatic chains in positions 2 and 7 (BTBT-CnOH) where the intralayer molecular stacking alternates between a classical and an inverted herringbone mode as a function of whether the alkyl sides chains have an even or an odd number of carbon atoms. This odd-even effect does not only affect the interlayer distance of the lamellar structures and the melting points, but also the electronic properties. The BTBT-CnOH odd series develops a classical herringbone pattern with edgeto-edge $S \cdots S$ interaction chains linked together by face-to-edge $S \cdots S$ interaction chains with two-dimensional mobility. However, the even series has only edge-to-edge interactions in an inverted herringbone organization and thus only a one-dimensional conducting character. 


\section{WILEY-VCH}

These two types of herringbone patterns have different Field Effect Transistor characteristics and mobilities, those of the odd members being systematically higher than their even neighbors. This is the first example of an odd-even effect impacting the electronic properties of an organic semiconductor.

\section{Introduction}

The odd-even effect in organic chemistry is a widely occurring phenomenon which can be defined as the dependency of structural, chemical and/or physical properties on the odd or even number of structural units in a molecule. Alternating solid-state packings as a function of whether the alkyl side chains have an even or odd number of carbon atoms and their influence on notably the melting point have indeed been observed in $n$-alkane and end-substituted $n$ alkanes. ${ }^{[1,2]}$ This odd-even effect has been in particular studied in organic $n$-alkane selfassembled monolayers $^{[3,4]}$ and has been observed for electronic ${ }^{[5]}$, frictional ${ }^{[6]}$ and electrochemical properties. ${ }^{[7]}$ Although crystal packing alternations for odd and even members in a particular series have been shown to be present for a wide range of organic materials, little attention has been paid to the odd-even effect in organic semiconductors. The devices of organic electronics are based on $\pi$-conjugated molecules capable of selfassembling in 1D, 2D or even 3D and controlling their organization allows to fine-tune their electronic properties and thus to optimize the resulting devices. ${ }^{[8]}$ With this objective, $\pi$ conjugated chromophores have been functionalized by alkyl chains and steric bulky moieties not only to improve their solubility ${ }^{[9]}$ but also to regulate their supramolecular organization and progress towards the control of performances of the resulting devices. ${ }^{[10,11]}$ In most cases the aliphatic parts are simple alkyl chains or aromatic groups introducing weak van der Waals, $\pi$-stacking or sulfur-sulfur interactions. These alkyl chains regulate many aspects of the solid- 


\section{WILEY-VCH}

state packing and are, in turn, indirectly responsible for the electronic and optical properties of the resulting organic semiconductor materials. So far, how these moieties govern such characteristics is not fully understood. In order to optimize the performances of the devices the length of their solubilizing aliphatic substituents is varied until an optimum is found. ${ }^{[12]}$ Until now, no rules could be established to predict the optimum of the number of $\mathrm{CH}_{2}$ units.

In a couple of studies odd- and even-length side chain materials with $\pi$-bonded units were synthesized but the possibility of an optoelectronic property alternation was overlooked or not

reported. For example, recently, Burnett et al. reported an odd-even effect ${ }^{[13]}$ on the properties of a number of physicochemical properties in a series of (2,5-bis(3-alkylthiophen-2-yl) thieno[3,2-b]thiophenes; BTTT) monomers with odd and even numbered alkyl side chains. In this case, the melting point was found to depend on the odd or even number of $\mathrm{CH}_{2}$ groups in the alkyl side chains, which could be correlated to different packing modes for BTTT with odd or even-numbered side chains. Another widely studied system is based on $\pi$-extended [1]benzothieno[3,2-b][1]benzothiophene (BTBT) containing aromatic compounds, which are known for their high charge carriers mobilities and perform as such among the best organic semiconductors for the field effect transistor application. ${ }^{[9,11,14]}$ Ebata et al. ${ }^{[9]}$ reported the synthesis, the characterization and the fabrication of field effect transistors (OFET) of a series of dialkyl[1]benzothieno[3,2-b][1]benzothiophenes (BTBT-Cn, Scheme 1) with n = 5-14 (where $n$ is the number of methylene units in the alkyl side chains) without studying the oddeven effect. They observed that the characteristic interlayer distance (the " $d$-spacing") depends on the length of the alkyl chains with larger $d$-spacings for longer alkyl chains. They concluded that all the derivatives have similar molecular packings, but only the crystal structure of the BTBT-C12 was solved and erroneously attributed to all the other molecules. However, using their provided structural information, Burnett et al. ${ }^{[13]}$ demonstrated that the out-of-plane d-spacing of the thin BTBT-Cn films was following an odd-even effect as 


\section{WILEY-VCH}

shown in Fig. S45 suggesting that the molecular packing was after all not similar for all members of the BTBT-Cn series without providing a detailed structural evidence.

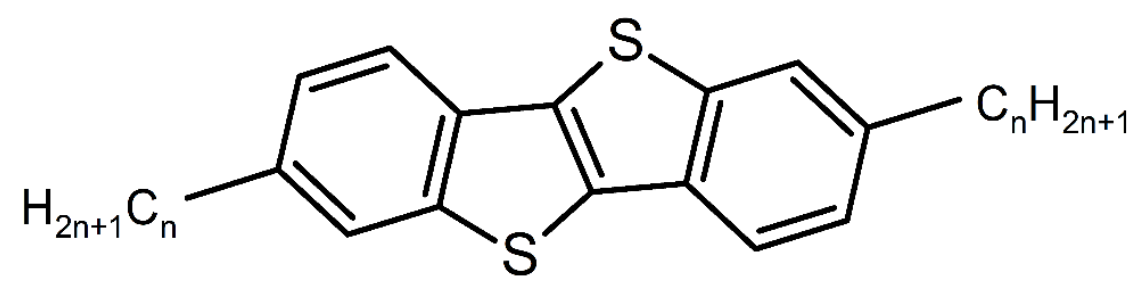

\section{BTBT-C $n$ n=5-14}

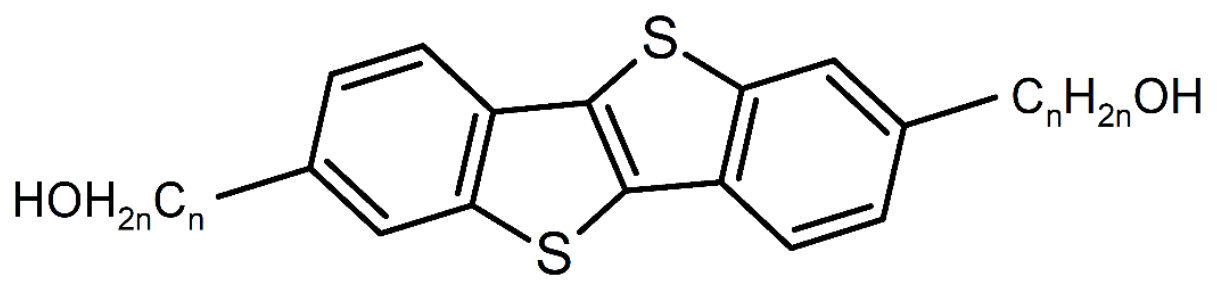

BTBT-C $n$ OH $n=5-10$

Scheme 1. Chemical schemes of BTBT-Cn and BTBT-CnOH.

Here we report a complementary study of the BTBT-Cn series, focusing mainly on the correlation between the molecular packing and the device characteristics as a function of the number of $\mathrm{CH}_{2}$ groups. We carried out single-crystal X-ray structure analyses for the not yet reported members of the BTBT-Cn series, i.e. for $n=6$, 7, and 9. However, since we did not find a real evidence of an impact of an odd-even effect of the structural organization on the optoelectronic properties of this family, we turned our attention to the BTBT-CnOH series of which we already reported the structures of the $n=4^{[15]}$ and $n=6^{[16,17]}$ members and the charge transport properties of the $n=6$ member. ${ }^{[16]}$ The $\mathrm{C}_{n} \mathrm{OH}$ chains are as in BTBT-Cn attached to 


\section{WILEY-VCH}

the 2 and 7 positions of the BTBT core (Scheme 1). In this study we report and compare the single-crystal structures of all $n=5-10$ members of the BTBT-CnOH series and we show the existence of a remarkable odd-even effect related to the orientation of the BTBT cores. We also report the optical properties and the FET mobilities of the devices of the complete series and correlate the structural features to these characteristics.

\section{Results and discussion}

\subsection{The BTBT-Cn series}

As already mentioned, all members of the BTBT-Cn series self-organize in a lamellar structure and by analogy with the X-ray structure of the BTBT-C12, the organization of their BTBT cores in a lamella was considered to be a herringbone arrangement $[7,14] .{ }^{[9,18]}$ This was confirmed by Izawa et al. ${ }^{[10]}$ who described and compared three crystal structures of the series (BTBT-C8, BTBT-C10 and BTBT-C12). Other BTBT-Cn structures, including odd members, have been studied recently ${ }^{[19]}$ and we complete the series in this study. The crystal structure of the $n=11$ member could not be obtained. Table 1 gives an overview of the cell parameters and space group symmetries of all members. 


\section{WILEY-VCH}
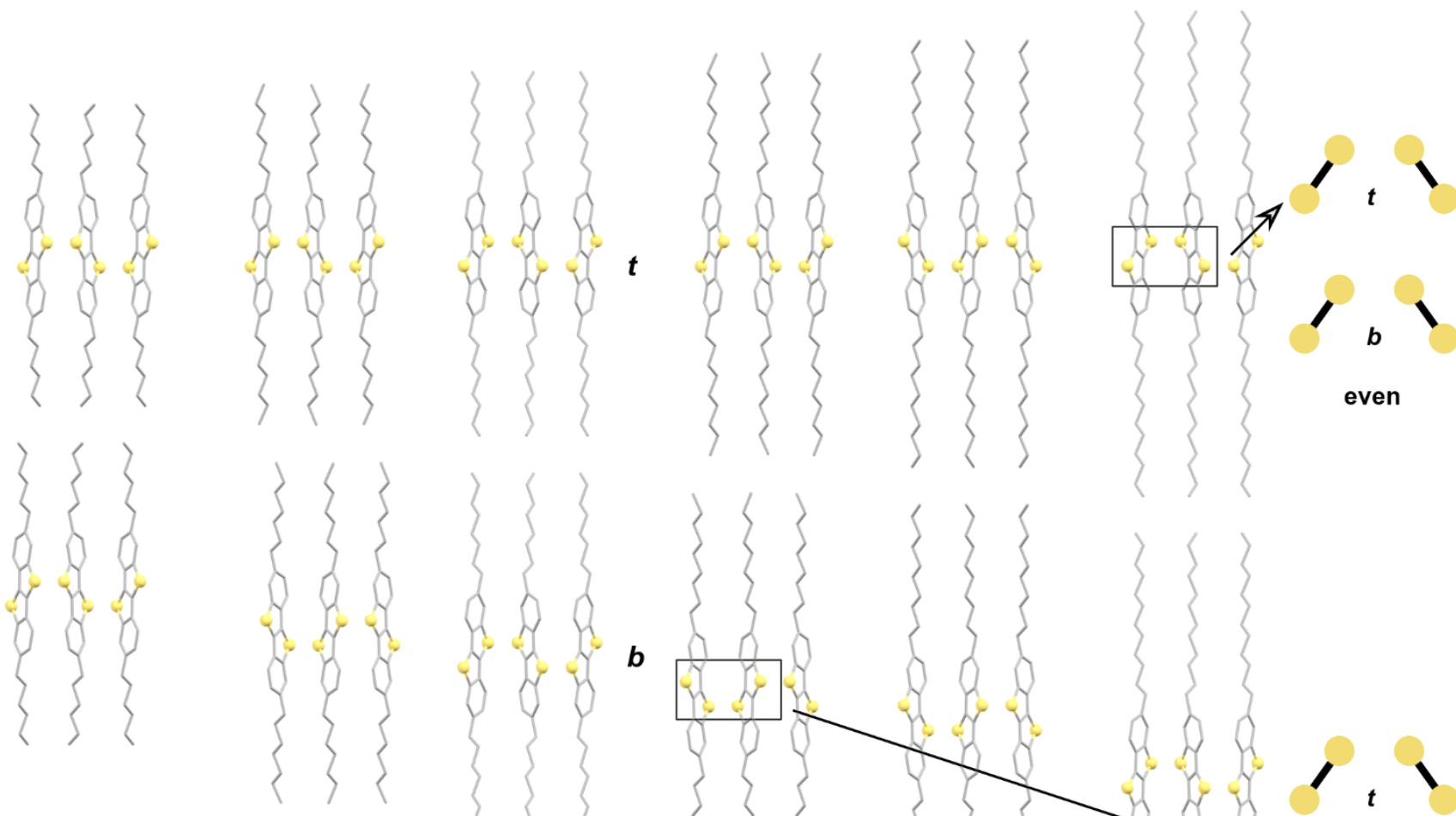

BTBT-C6

BTBT-C7

BTBT-C8

BTBT-C9

BTBT-C10

BTBT-C12

Figure 1. Projections of the structures of BTBT-Cn for $n=6,7,8,9,10,12$ along the $a$-axis. The crystal structure of the $n=11$ member could not be obtained. The $b$-axis points in all cases to the right and the $c$-axis downwards. Hydrogen atoms are omitted in all figures for clarity. The schemes at the right represent the vertical stacking modes of S-S pairs between the top $(t)$ and bottom $(b)$ molecular layers for the even and the odd members, respectively.

The compounds BTBT-C2-BTBT-C5 do not possess a favorable stacking of the BTBT cores making semiconductor behavior impossible due to the absence of close $\mathrm{S} \cdots \mathrm{S}$ contacts. All

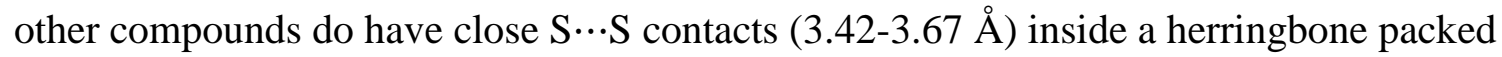
layer parallel to the $a b$-plane, facilitating two-dimensional carrier transport in this direction. The herringbone layers themselves are packed along the crystallographic $c$-axis, but not in the same way for the odd and even members. The unit cell parameters for all compounds $n=6-12$ follow the same trend, i.e. an $a$-parameter around 5.8-5.9 $\AA$, a $b$-parameter around 7.6-7.9 $\AA$, 


\section{WILEY-VCH}

a $c$-parameter which depends clearly on the length of the alkyl chain and a $\beta$-angle close to 90 . All structures, except BTBT-C6, crystallize in space group $n^{\circ} 14$, which in its standard setting is $P 2_{1} / c^{[20]}$, but are reported in the three different settings $P 2_{1} / c, P 2_{1} / a$, and $P 2_{1} / n$. This means that the structures - without considering the different lengths of the alkyl chains are not isostructural, but do display different packings, except those reported in the same setting, i.e. the even members $n=8,10,12$, which are indeed isostructural and which include the $n=6$ member that is a slightly triclinically distorted variant of the structures of the other even members. Figure 1 shows the herringbone packed structures of all compounds with $n>=6$ projected along the $a$ and $c$-axes. The projection along the a-axis shows clearly the different packing of the layers for odd and even members: whereas for all even numbers the two sulfur atoms of the BTBT core in different layers are arranged in a parallel fashion with respect to each other, they are anti-parallel for the odd members. In addition, the end-methyl groups that are opposite to each other adopt a staggered conformation which is much more pronounced for the odd members than for the even members. In the projection along the $a$-axis this shows up as a larger lateral shift in the $b$-direction for the odd members than for the even members.

Table 1. Cell parameters $a, b, c, \alpha, \beta, \gamma$, and cell volume $V$ of all BTBT-Cn compounds for which a structure determination from singe crystal X-ray data was reported.

\begin{tabular}{|c|c|c|c|c|c|c|c|c|c|c|c|}
\hline \multirow[t]{2}{*}{ BTBT-Cn } & \multicolumn{7}{|c|}{ Cell parameters } & \multirow{2}{*}{$\begin{array}{l}\rho(\mathrm{gcm} \\
\left.{ }^{3}\right)\end{array}$} & \multirow{2}{*}{$\begin{array}{l}\text { space } \\
\text { group } \\
\text { symmetry }\end{array}$} & \multirow{2}{*}{$\begin{array}{l}T \\
(\mathrm{~K})\end{array}$} & \multirow{2}{*}{$\begin{array}{l}\text { Reference/CSD } \\
\text { refcode }\end{array}$} \\
\hline & $a$ & $b$ & $c$ & $\alpha$ & $\beta$ & $\gamma$ & $V$ & & & & \\
\hline BTBT-C2 & 7.827 & 8.340 & 12.326 & 90 & 108.84 & 90 & 761.53 & 1.293 & $P 2_{1} / \mathrm{a}$ & 300 & 15/LAWMEE \\
\hline BTBT-C3 & 4.683 & 7.453 & 12.480 & 76.95 & 85.20 & 84.36 & 421.407 & 1.279 & $P-1$ & 300 & 15/LAWMII \\
\hline BTBT-C4 & 4.643 & 7.652 & 13.444 & 86.1 & 82.18 & 84.25 & 470.0779 & 1.245 & $P-1$ & 300 & 15/LAWMOO \\
\hline BTBT-C5 & 4.759 & 8.681 & 13.114 & 89.73 & 80.94 & 77.69 & 522.512 & 1.210 & $P-1$ & 300 & 15/LAWMUU \\
\hline BTBT-C6 & 5.9084 & 7.6673 & 24.6362 & 92.040 & 92.858 & 90.111 & 1113.96 & 1.218 & $P-1$ & 100 & This study \\
\hline BTBT-C7 & 5.8700 & 7.6160 & 54.982 & 90 & 91.19 & 90 & 2457.5 & 1.180 & $P 2_{1} / \mathrm{c}$ & 100 & This study \\
\hline
\end{tabular}


WILEY-VCH

\begin{tabular}{|l|l|l|l|l|l|l|l|l|l|l|l|}
\hline BTBT-C8 & 5.927 & 7.880 & 29.180 & 90 & 92.44 & 90 & 1361.606 & 1.134 & $P 2_{1} / \mathrm{a}$ & 300 & $8 \mathrm{a} /$ YOKBIK \\
\hline BTBT-C9 & 5.8675 & 7.5471 & 63.937 & 90 & 91.65 & 90 & 2830.1 & 1.157 & $P 2_{1} / \mathrm{n}$ & 100 & This study \\
\hline $\begin{array}{l}\text { BTBT- } \\
\text { C10 }\end{array}$ & 5.923 & 7.838 & 33.783 & 90 & 93.73 & 90 & 1565.041 & 1.105 & $P 2_{1} / \mathrm{a}$ & 300 & $8 \mathrm{a} / \mathrm{YOKBOQ}$ \\
\hline $\begin{array}{l}\text { BTBT- } \\
\text { C12 }\end{array}$ & 5.864 & 7.740 & 37.910 & 90 & 90.59 & 90 & 1720.544 & 1.114 & $P 2_{1} / \mathrm{a}$ & 300 & $7 /$ PIVBAY \\
\hline
\end{tabular}

Notes: cell lengths in $\AA$; cell angles in ${ }^{\circ}$, cell volume in $\AA^{3}$. The CSD refcode is the identifier in the Cambridge Structural Database.

The different stacking of alkyl containing compounds with odd and even numbers of methylene groups is a known phenomenon and related to the lower possibility of alkyl chains with an odd number of methylene units to pack in a compact way in the zones where the methyl groups are opposite to each other than alkyl chains with an even number of methylene units. ${ }^{[2]}$

It is possible to quantify the compactness of the packing of adjacent molecular BTBT-Cn layers by calculating the fraction of the 'free' or 'occupiable' volume relative to the unit cell volume by using a virtual spherical probe. ${ }^{[21]}$ This computational procedure is usually employed for the calculation of porosity or solvent-accessible volumes, where probe radii between 1.2 and $1.6 \AA$ are commonly employed. ${ }^{[22]}$ In this case there is not any solvent accessible volume, since all BTBT-Cn compounds are strictly dense, but it appears that with a lower probe radius - here $0.95 \AA$ - than the radius used for common intruding species useful information can be obtained about the compactness of the packing of adjacent molecular BTBT-Cn layers and thus indirectly the Van der Waals interaction between the methyl contact planes. The virtual spherical probe method has the advantage that the subatomic-sized free volumes can be located within space, which is not the case for other methods that calculate the compactness of a crystal structure, such as the Kitajgorodskii packing index method. ${ }^{[23]}$ Here MERCURY ${ }^{[24]}$ was used for the calculations using a grid of $0.1 \AA$ : all subatomic free volumes are indeed found in the methyl contact layer between opposite alkyl chains (Fig. S48-S53). Interestingly, for the structures $n=2,3,4,5$ which also 


\section{WILEY-VCH}

pack layered-wise, but with a different arrangement of opposite alkyl chains, the subatomic-

sized free volumes are not found in the zones between the terminal methyl groups, but in between the alkyl chains within the layers. Figure 2Erreur ! Source du renvoi

introuvable.a gives the results of the calculations for $n=6-12$ showing a clear odd-even effect, and confirming Boese's hypothesis ${ }^{[2]}$ related to the lower compactness of the packing of adjacent molecular layers for odd-membered $n$-alkanes compared to even-membered $n$ alkanes.
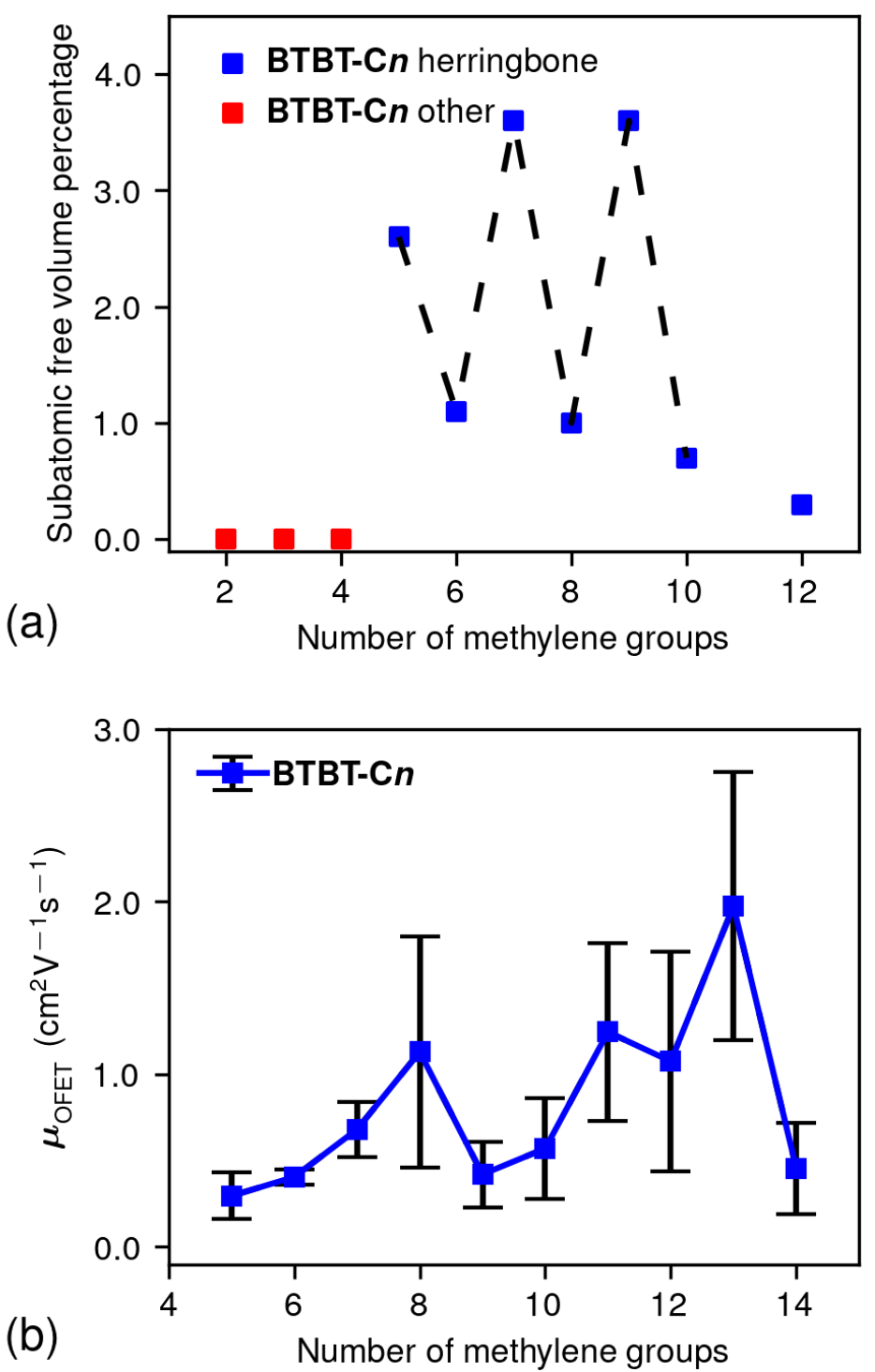

Figure 2 (a) \% free volume per unit-cell volume versus number of methylene groups in BTBT-Cn for a probe radius of $0.95 \AA$ and a grid-spacing of $0.1 \AA$. The blue markers are for the structures that stack according to the herringbone mode, whereas the red markers are for structures with other stackings. It is noted that the crystal structure of the $n=11$ member could 


\section{WILEY-VCH}

not be obtained. A larger \% free volume value means a less compact inter-layer space. (b) Mobilities of OFET BTBT-Cn devices evaluated under ambient conditions without any precautions to eliminate air and moisture produced from Table S1 and ref ${ }^{[9]}$. Experimental values from 10 different devices are found within the error bars.

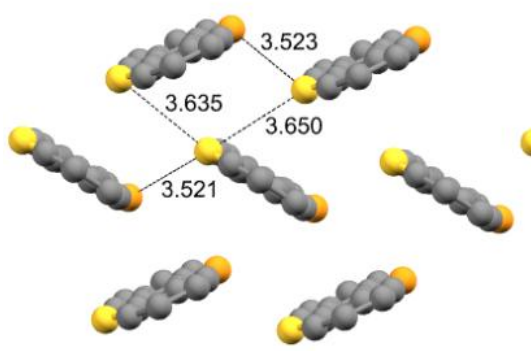

BTBT-C6

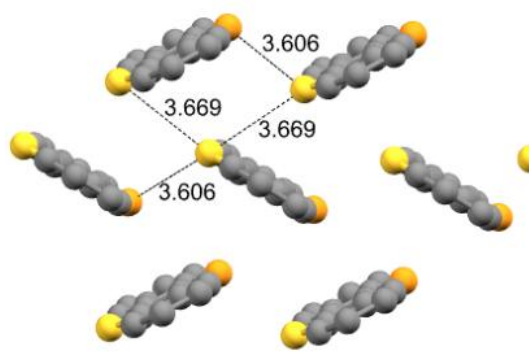

BTBT-C8

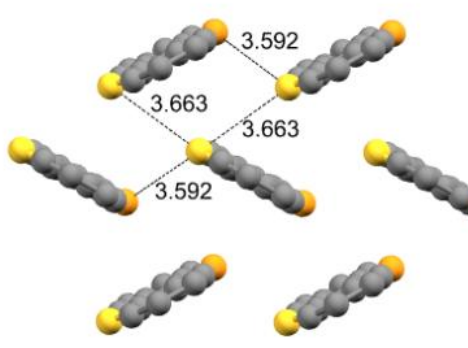

BTBT-C10

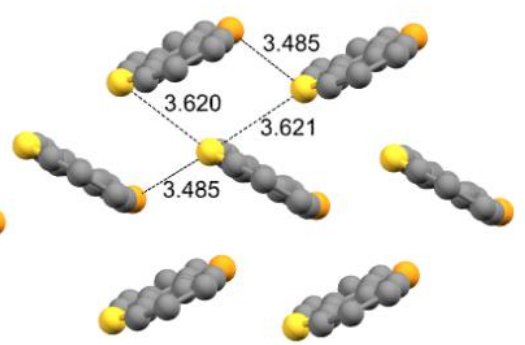

BTBT-C7

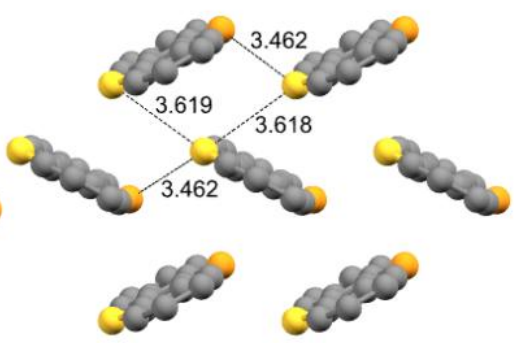

BTBT-C9

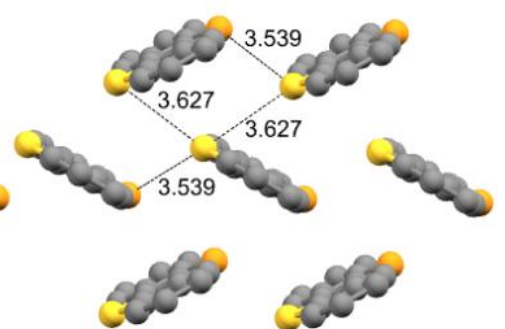

BTBT-C12

Figure 3. Projections of the BTBT-Cn structures for $n=6,7,8,9,10,12$ along the $c$-axis. It is noted that the crystal structure of the $n=11$ member could not be obtained. For clarity, hydrogen atoms and alkyl chains are omitted in all sub-figures. The yellow sulfur atoms are in the foreground, whereas the orange sulfur atoms are in the background. They all present the classical herringbone pattern.

The projection along the $c$-axis in Figure 3 shows that all BTBT-Cn structures have the same type of BTBT core layer with the same orientation of the BTBT cores within herringbonemode packing but without any odd-even effect. This stacking will be denoted as the classical herringbone orientation in the following. ${ }^{[18,25]}$ There exist two different intermolecular $S \cdots S$ 


\section{WILEY-VCH}

contacts shorter than the Van der Waals diameter of sulfur atoms $(3.70 \AA)$ which connect the BTBT cores in an edge-to-edge manner or in a face-to-edge manner. The edge-to-edge $S \cdots S$ interaction chains have a short S $\cdots \mathrm{S}$ distance of the order of $3.55 \AA$ whereas the face-to-edge interaction have longer $\mathrm{S} \cdots \mathrm{S}$ distances of the order of $3.65 \AA$, both interactions conferring a two-dimensional character to the crystalline organization and a hence a 2D mobility.

Figure $2 \mathrm{~b}$ displays reported BTBT-Cn mobilities, $\mu_{\mathrm{OFET}}$, according to the data available in the literature (Table S1). Although no pronounced dependence of $\mu_{\mathrm{OFET}}$ on alkyl group length was observed, BTBT-Cns with longer alkyl group tend to exhibit higher mobility values than the derivatives with shorter alkyl groups. For example, $\mu_{\mathrm{OFET}}$ for the well-known BTBT-C8 is higher by approximately on order of magnitude than that for BTBT-C5 but no odd-even discrimination can be observed, which is not surprising since the orientation of the BTBT cores remains identical throughout the series. The best mobilities among BTBT-Cn derivatives are for BTBT-C13 around $2.0 \mathrm{~cm}^{2} \mathrm{~V}^{-1} \mathrm{~s}^{-1}[9]$ but recently devices with record-high mobilities at $17.2 \mathrm{~cm}^{2} \mathrm{~V}^{-1} \mathrm{~s}^{-1}$ were reported, ${ }^{[26]}$ close to the golden standard value of single crystal rubrene-based devices. The atomic structure of BTBT-C13 has not, however, yet been reported.

\subsection{The BTBT-CnOH series}

Despite the fact that an odd-even effect is obviously present in the $d$-spacings of the lamellar BTBT-Cn phases, no effect on the opto-electronic properties of the resulting devices can be identified because of the identical intra-layer structures. The supramolecular reason behind this behavior can most probably be found by considering the strength of the different interactions involved in the self-assembly of the supramolecular material. We can reasonably propose that the interlayer herringbone pattern is firstly formed involving $\sigma-\pi$ stacking and the $\mathrm{S} \cdots \mathrm{S}$ interactions and that the resulting semiconducting layers then self-assemble into a 


\section{WILEY-VCH}

lamellar phase through van der Waals interactions between opposite alkyl chains, since the former interactions are stronger than the latter. Therefore, the organization of the $\pi$ conjugated cores is not controlled by the odd-even effect originating from the aliphatic insulating part.

A literature survey concerning compounds undergoing an odd-even effect influencing their physicochemical and electronic properties, ${ }^{[3]}$ reveals the requirement for the effect to be present to have a strong interaction at the end of aliphatic chains, either by a covalent bond in the case of monolayers $\left(\mathrm{Ag}-\mathrm{CH}_{3}\left(\mathrm{CH}_{2}\right)_{\mathrm{n}} \mathrm{SH},{ }^{[27]} \mathrm{Au}-\mathrm{CH}_{3}\left(\mathrm{CH}_{2}\right)_{\mathrm{n}} \mathrm{SH}^{[28]}\right)$ or by a hydrogen bond interaction in the case of lamellar compounds such as $\mathrm{CH}_{3}\left(\mathrm{CH}_{2}\right)_{\mathrm{n}-2} \mathrm{COOH}$, ${ }^{\text {[29] }}$ $\mathrm{HO}\left(\mathrm{CH}_{2}\right)_{\mathrm{n}-1} \mathrm{CH}_{3}$, or $\mathrm{HO}\left(\mathrm{CH}_{2}\right)_{\mathrm{n}-1} \mathrm{COOH} .{ }^{[30]}$ In this case, the odd-even modification of the orientation of the end group is directed by the covalent or non-covalent bonds at the end of the alkyl chain and could also influence the supramolecular assembly as a function of the odd or even number of alkyl spacers. ${ }^{[31]}$

In this context we decided to add a structure directing agent at both ends of the alkyl chains of BTBT-Cn and for that purpose the $\mathrm{OH}$ function was selected, because the resulting hydrogen bond should be strong enough to promote the formation of a hydrogen bond network and weak enough to preserve the solubility of this BTBT-CnOH series (Scheme 1). Their synthesis involves a Friedel and Kraft addition of two $\mathrm{Br}\left(\mathrm{CH}_{2}\right)_{\mathrm{n}} \mathrm{COCl}$ on position 2 and 7 of the BTBT core (see supporting information), followed by the conversion of the bromines into acetates. Finally, the use of hydrazine hydrate can conjointly reduce the carbonyl functions located in position 2 and 7 and realize the hydrolysis of the acetate to generate the hydroxy function.

Out-of-plane X-ray diffraction realized in Bragg-Brentano configuration on thin BTBT$\mathrm{CnOH}$ films vacuum evaporated on silicon wafers reveal that all members are self-organized according to a lamellar structure developing perpendicular to the substrate with characteristic 


\section{WILEY-VCH}

$d$-spacings connected to the interlayer spacing that increase with the length of the alkyl chains

(Figure 4a). As in the case of the BTBT-Cn compounds, a slight crystal-packing odd-even alternation is observed (Figure 4). The much more effective cohesion between the layers in BTBT-CnOH is demonstrated by their much smaller interlayer-spacing compared to the BTBT-Cn compound with the same number of spacers, i.e. BTBT-C $(\boldsymbol{n}+\mathbf{1})$. This difference increases steadily from $1.4 \AA$ for the pair BTBT-C5OH/BTBT-C6 (23.1/24.5 $\AA$ ) to $2.6 \AA$ for the pair BTBT-C10OH/BTBT-C11 (33.3/35.9 $\AA$ ). A small fraction of these differences is caused by the shorter $\mathrm{C}$-O distance $(1.4 \AA$ ) compared to the $\mathrm{C}$ - $\mathrm{C}$ distance $(1.5 \AA)$, but the main contribution comes from the efficient $\mathrm{OH} \cdots \mathrm{O}$ bonding network between adjacent molecular BTBT-CnOH layers. The much reported melting point alternation for compounds containing long alkyl chains is equally well confirmed for the BTBT-CnOH series (Fig. S44).
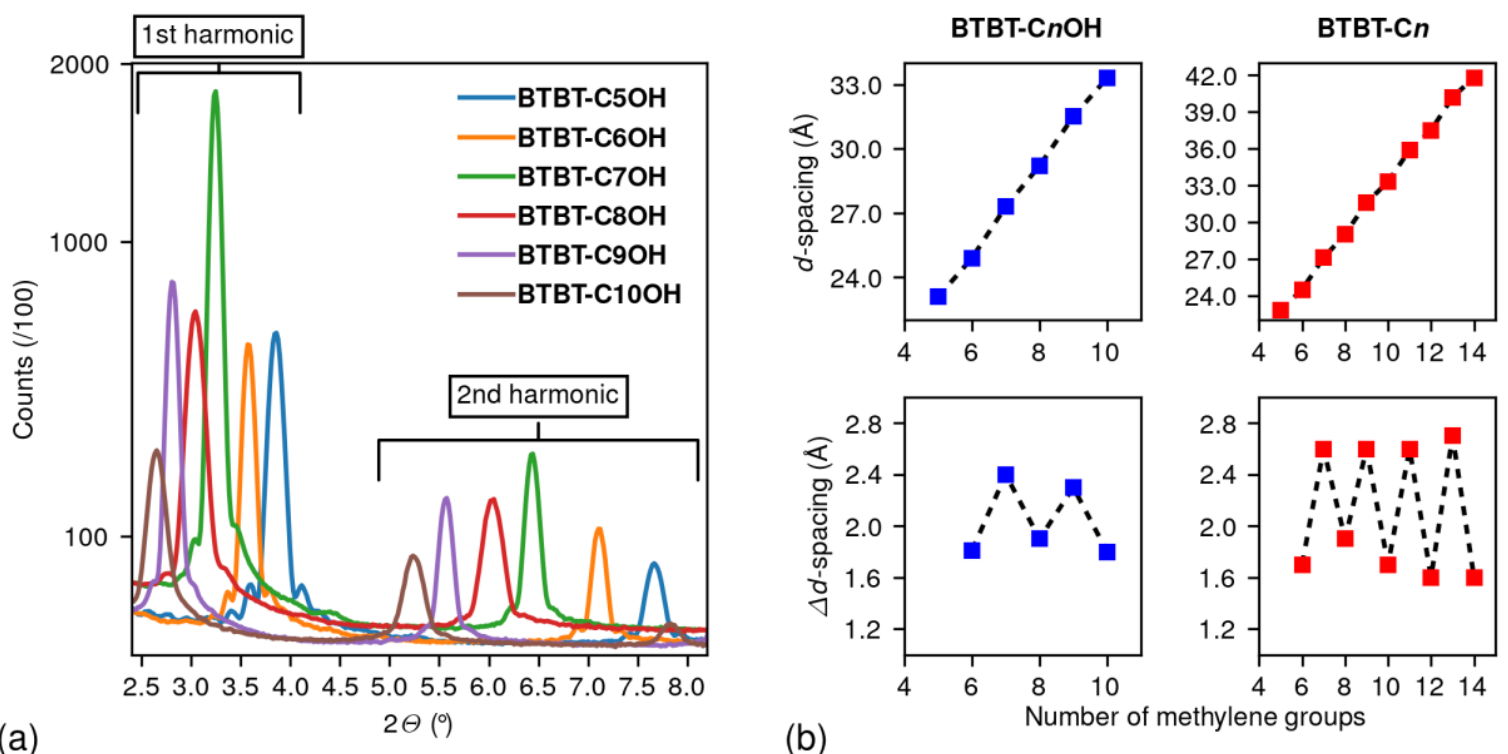

Figure 4 (a) Out-of-plane XRD realized at ambient conditions on thin films of the BTBTCnOH series with systematically varied side-chain length evaporated on silicon wafers (this work). The first group of peaks corresponds to the first reflection harmonic related to the characteristic interlayer distance (the " $d$-spacing"), whereas the second group corresponds to the second harmonic of the same reflection peak. The vertical scale is a square-root scale. (b) Out-of-plane $d$-spacings of the BTBT-CnOH obtained from the out-of-plane XRD. For comparison the $d$-spacings reported for BTBT-Cn (reproduced with permission from Chem. Mater. 2019, 31, 6900-6907 Copyright (2019) American Chemical Society) are given here as 


\section{WILEY-VCH}

well. The difference in $d$-spacing between successive members in the lower part of the figure illustrates the odd-even effect for both compounds.

The single-crystal structure of BTBT-C6OH was already reported before by us ${ }^{[16,17]}$ and by comparison with the structure of BTBT-C8 it could be inferred that in BTBT-C6OH, BTBT half of the cores are rotated by $180^{\circ}$ around their long axis in an inverted herringbone organization with only one type of $S \cdots S$ contact, viz. edge-to-edge $S \cdots S$ contacts with $S \cdots S$ distance of $3.545 \AA$, whereas face-to-edge $S \cdots S$ contacts are absent in this case. As a result, the structure of BTBT-C6OH has a more one-dimensional character than that of BTBT-C8. This was confirmed by the calculation of transfer integrals yielding strong interactions ( 65 $\mathrm{meV}$ ) between BTBT cores in one direction of the herringbone layer and very low interactions in the other direction, ${ }^{[16]}$ in contrast with BTBT-C8 which has a more homogeneous distribution of the transfer integrals in the layer.

We succeeded in growing single crystals for the $n=4,5,7,8,9$, and 10 members of the BTBTCnOH series. The $n=4^{[15]}$ member appeared to have an alternative stacking mode with a considerably denser structure, as have the low $n$-members of the BTBT-Cn series. The $n=5$ member crystallized in two polymorphic forms, of which one possesses the classical herringbone pattern. The $n=5,6,7,8,9,10$ members showed a remarkable odd-even effect in terms of diffraction quality, the even members displaying excellent diffracting efficiency, whereas the odd members had poor diffracting power, making the use of synchrotron radiation indispensable for the resolution of their atomic structures. All compounds crystallized as platelets, but the odd $n=5,7,9$ members were much thinner than the $n=6,8,10$ members. The lower probability to obtain crystals for organic compounds suitable to be analyzed by X-ray diffraction with an odd number of methylene spacers was noticed in several studies. ${ }^{[32]}$ 


\section{WILEY-VCH}

We have tried to obtain X-ray data for all compounds at ambient conditions, since these are the usual conditions for the functional devices made from these compounds. For some of the structures the data collected at room temperature did not result, however, in acceptable chemical structures and therefore data were collected at lower temperatures.

Table 2 gathers the cell parameters and the space group symmetries of all members. The $n=6,8,10$ members crystallize in the same monoclinic space group $C 2 / c$ and are isostructural apart from the different alkyl chain lengths. The same holds for the $n=7$ and 9 members in the triclinic space group $P-1$, but the $n=5$ member has space group $P 2_{1} / c$ with nevertheless the same packing mode as the two odd triclinic members. The structure of the $n=5$ member was also measured at low temperatures to ascertain that no phase transition takes place; it was indeed found that the space group at $100 \mathrm{~K}$ is also $P 2{ }_{1} / c$ [33] and its atomic arrangement isostructural with the room temperature structure. Whereas this study focusses on the inter and intramolecular packing mode which is mostly independent of temperature when there are no phase transitions, intermolecular interactions may be more or less dependent on temperature. This has been previously demonstrated by us for $n=4,5,6[15,17,33]$ and will be the topic of a forthcoming study.

Table 2. Cell parameters $a, b, c, \alpha, \beta, \gamma$, and cell volume $V$ of all BTBT-CnOH compounds for which a structure determination from single crystal X-ray data was reported.

\begin{tabular}{|c|c|c|c|c|c|c|c|c|c|c|c|}
\hline \multirow{2}{*}{$\begin{array}{l}\text { BTBT- } \\
\mathrm{CnOH}\end{array}$} & \multicolumn{7}{|c|}{ Cell parameters } & \multirow{2}{*}{$\begin{array}{l}\rho \\
\left(\mathrm{gcm}^{-}\right. \\
\left.{ }^{3}\right)\end{array}$} & \multirow{2}{*}{$\begin{array}{l}\text { space } \\
\text { group } \\
\text { symmetry }\end{array}$} & \multirow{2}{*}{$\begin{array}{l}T \\
(\mathrm{~K})\end{array}$} & \multirow{2}{*}{$\begin{array}{l}\text { Reference/CSD } \\
\text { refcode }\end{array}$} \\
\hline & $a$ & $b$ & $c$ & $\alpha$ & $\beta$ & $\gamma$ & $\bar{V}$ & & & & \\
\hline $\begin{array}{l}\text { BTBT- } \\
\text { C4OH }\end{array}$ & 13.748 & 5.072 & 14.477 & 90 & 108.402 & 90 & 957.9 & 1.333 & $P 2_{1} / \mathrm{c}$ & 300 & ${ }^{[15]} /$ TUVHIE20 \\
\hline BTBT- & 5.869 & 47.39 & 7.892 & 90 & 90.390 & 90 & 2195.0 & 1.249 & $P 2_{1} / \mathrm{c}$ & 275 & This study \\
\hline
\end{tabular}


WILEY-VCH

\begin{tabular}{|l|l|l|l|l|l|l|l|l|l|l|l|}
\hline C5OH & & & & & & & & & & & \\
\hline $\begin{array}{l}\text { BTBT- } \\
\text { C6OH }\end{array}$ & 51.3186 & 5.5820 & 8.4325 & 90 & 102.270 & 90 & 2360.40 & 1.240 & $C 2 / c$ & 300 & ${ }^{[16 / \text { UROBUA11 }}$ \\
\hline $\begin{array}{l}\text { BTBT- } \\
\text { C7OH }\end{array}$ & 5.8484 & 7.7669 & 27.7880 & 87.525 & 89.047 & 89.998 & 1260.89 & 1.180 & $P-1$ & 173 & This study \\
\hline $\begin{array}{l}\text { BTBT- } \\
\text { C8OH }\end{array}$ & 58.5920 & 5.2836 & 8.9316 & 90 & 90.501 & 90 & 2764.9 & 1.193 & $C 2 / c$ & 300 & This study \\
\hline $\begin{array}{l}\text { BTBT- } \\
\text { C9OH }\end{array}$ & 5.8318 & 7.6036 & 32.5337 & 88.313 & 86.120 & 89.911 & 1438.70 & 1.157 & $P-1$ & 100 & This study \\
\hline $\begin{array}{l}\text { BTBT- } \\
\text { C100H }\end{array}$ & 66.9510 & 5.1740 & 9.0401 & 90 & 94.640 & 90 & 3121.3 & 1.176 & $C 2 / c$ & 300 & This study \\
\hline
\end{tabular}

Notes: Cell lengths in $\AA$; cell angles in ${ }^{\circ}$, cell volume in $\AA^{3}$. The CSD refcode refers to the identifier in the Cambridge Structural Database. The cell parameters of BTBT-C6OH have been reported elsewhere ${ }^{[17]}$ in the equivalent setting $I 2 /$ a of space group $C 2 / c$. BTBT-C5OH has a polymorph whose structure is reported elsewhere. ${ }^{[33]}$

As already deduced from the out-of-plane XRD measured on evaporated thin films of BTBT-

$\mathrm{CnOH}$, all single crystal structures show a lamellar organization with an interlayer distance increasing with the number of methylene groups. (Figure 5). The $180^{\circ}$ rotation of one half of the BTBT cores is present for all even members within the BTBT-CnOH series, whereas the odd members have all the same relative BTBT orientations as the members in the BTBT-Cn series. As in the structures of BTBT-Cn, an odd-even alternation for the BTBT core stacking between adjacent molecular layers is found for BTBT-CnOH (Figure 5; stacking schemes on the right, $c f$ stacking schemes of BTBT-Cn in Figure 1), but not in the same way. The BTBT cores of the odd members of BTBT-CnOH stack in the same way as those of the even members of BTBT-Cn, i.e. an in phase positioning of the sulphur 'dimers' between the top $(t)$ layer and the bottom $(b)$ layer, but an out of phase orientation within the layer. In the structure of the even members of BTBT-CnOH the S-S dimer orientation is in phase both 


\section{WILEY-VCH}

within the layers and between the layers, whereas the odd members of BTBT-Cn have an out of phase orientation both within the layers and without the layers. These odd-even interlayer packing alternations for both the BTBT-Cn series and the BTBT-CnOH series have, however, no impact on the transport properties of these compounds, since they do not concern a structural alternation within the molecular layers where the transport takes place.

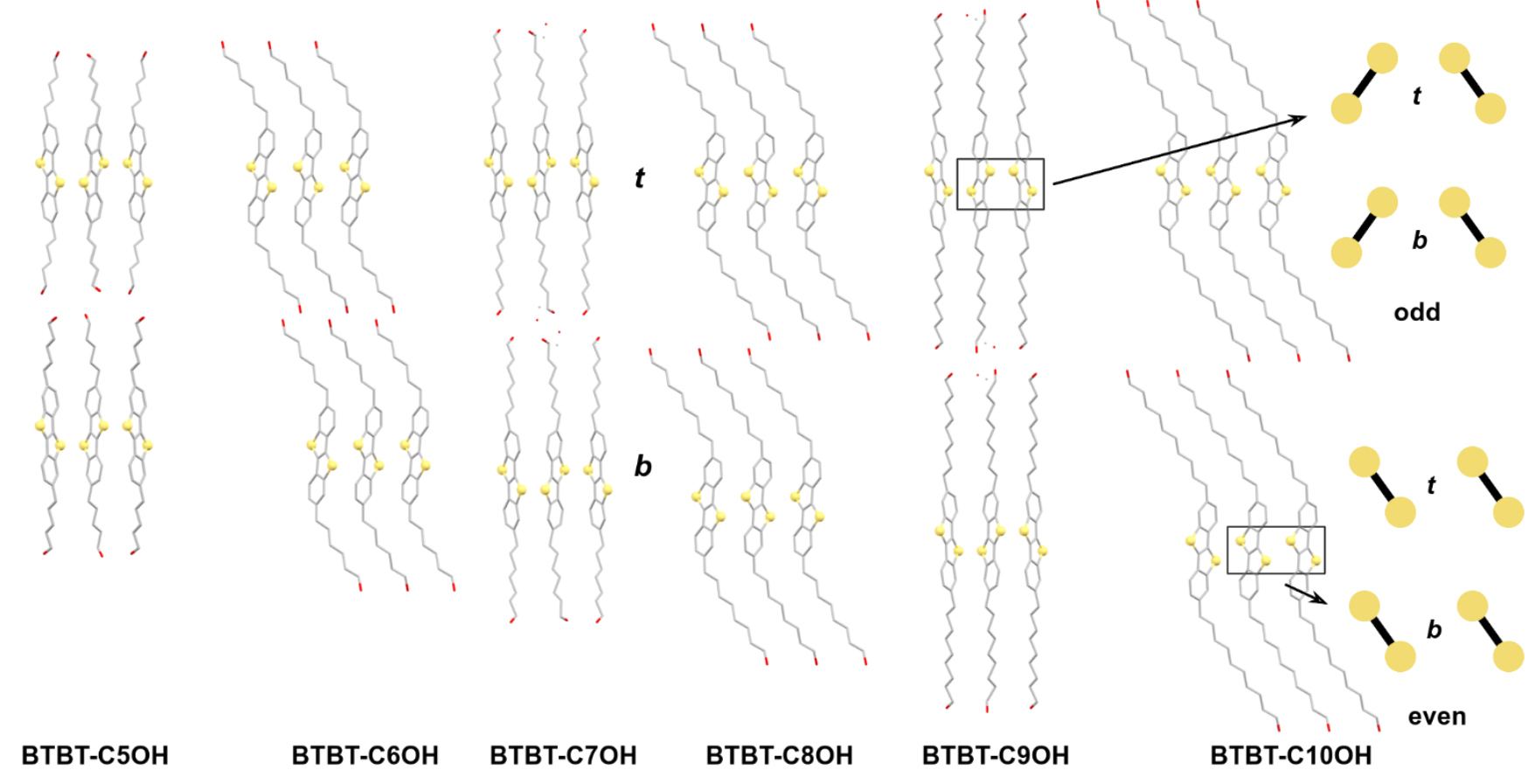

Figure 5. Projections of the BTBT-CnOH structures for $n=5,6,7,8,9,10$. Hydrogen atoms were omitted in all structures for clarity. The projections are in the same direction as in Figure 1, but because of the different space groups this is not always along the same crystallographic axis. The schemes at the right represent the vertical stacking modes of S-S pairs between the top $(t)$ and bottom $(b)$ molecular layers for the even and the odd members, respectively. 


\section{WILEY-VCH}

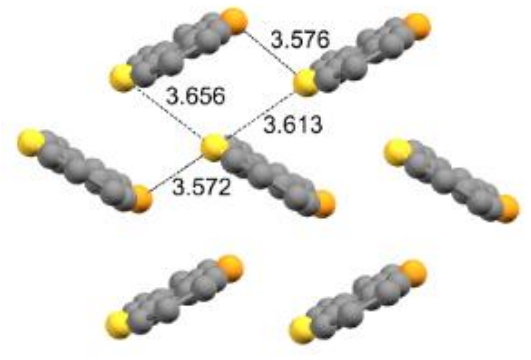

BTBT-C5OH

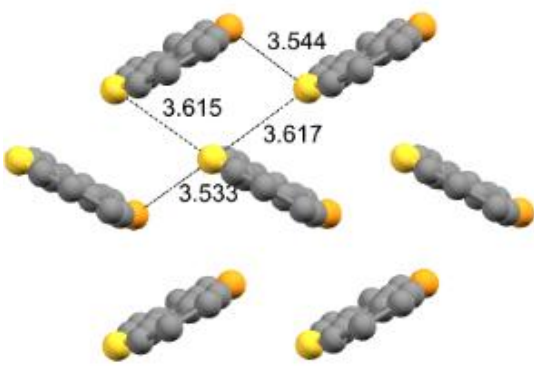

BTBT-C7OH

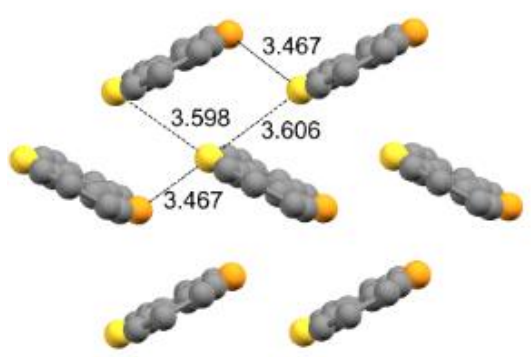

ВТВT-C9OH

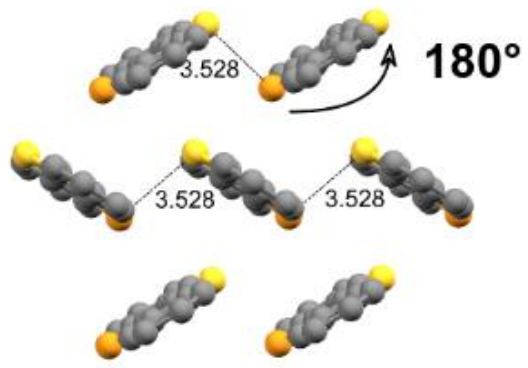

BTBT-C6OH

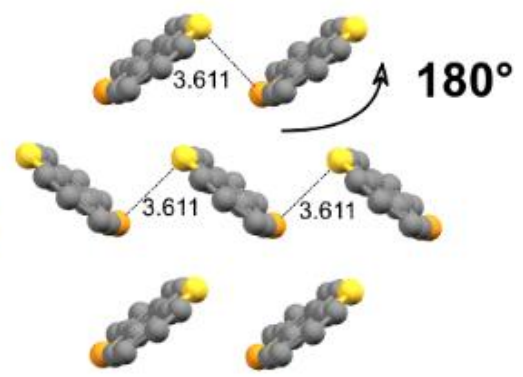

BTBT-C8OH

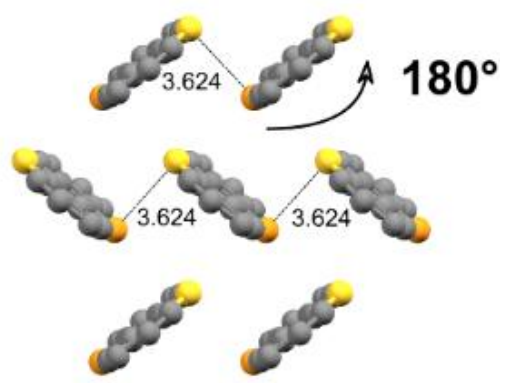

BTBT-C10OH

Figure 6. Classical (odd members) and inverted (even members) herringbone patterns in the structures of BTBT-CnOH. The yellow sulfur atoms are in the foreground, whereas the orange sulfur atoms are in the background. The BTBT moieties of the even members are rotated by $180^{\circ}$ with respect to those of the odd members.

The subatomic free volume between two adjacent molecular layers was calculated in the same way as for BTBT-Cn, where each layer in this case is bordered by hydroxyl moieties rather than methyl moieties as in the case of BTBT-Cn. The analysis for BTBT-CnOH, however, is more delicate, since the hydroxyl moieties are partially disordered over different positions, except in the case of BTBT-C5OH.

The hydrogen atoms of the terminate hydroxyl groups of the even members are disordered over two different positions, whereas the disorder for the odd members $n=7,9$ is more complicated, involving the complete terminate alcohol groups. In order to do the free-volume 


\section{WILEY-VCH}

calculation the structures were expanded to the $P 1$ space group and one hydrogen-bonded network was chosen, after which the analysis was carried out with the same parameters as for BTBT-Cn. Figure 7a shows the same tendency as for BTBT-Cn, i.e. a less compact hydroxy plane between adjacent molecular layers for the odd members than for the even members, and no free volume for the members that do not crystallize in a classical herringbone mode. This is rather surprising, since it could be expected that because of the even number of nonhydrogen atoms in the alcohol chain in BTBT-CnOH the odd members would have a denser Van der Waals region. It is probably the smaller volume of the $\mathrm{OH}$ group compared to the terminal $\mathrm{CH}_{3}$ group which makes that it is only the number of methylene groups that matter.

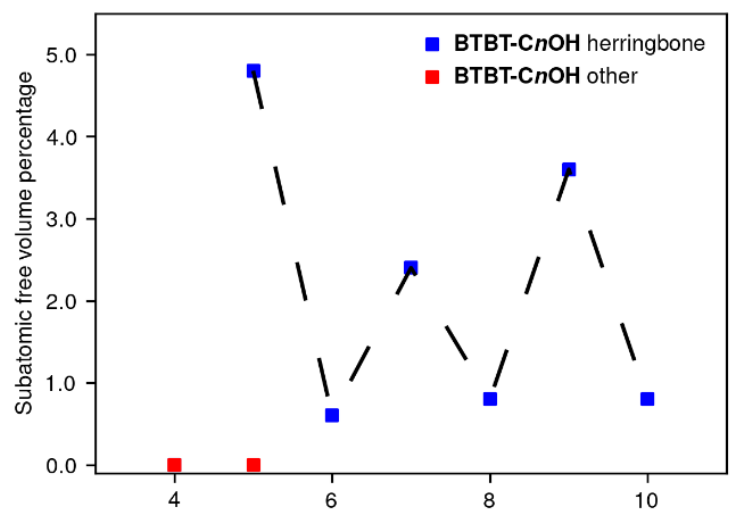

(a)

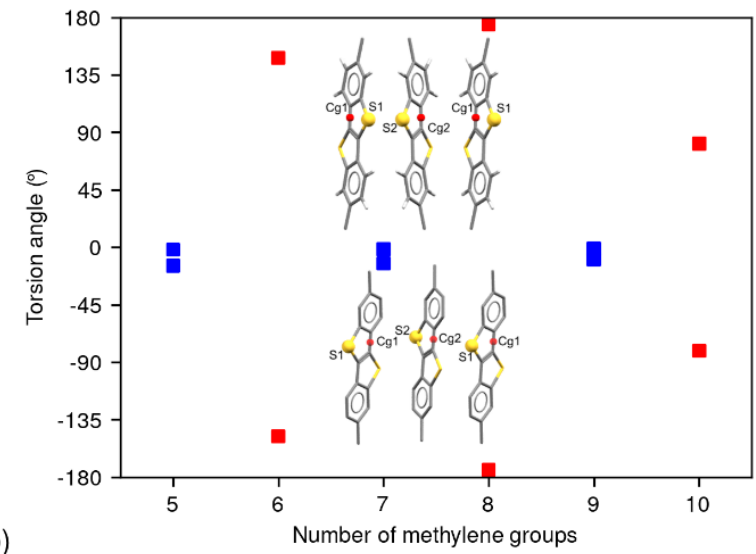

Figure 7 (a) \% free volume per unit-cell volume versus number of methylene groups in BTBT-CnOH for a probe radius of $0.95 \AA$ and a grid-spacing of $0.1 \AA$. The blue points are for the structures that stack according to the herringbone mode, whereas the red points are for structures with other stackings. (b) : Torsion angles $\mathrm{Cg} 1 \cdots \mathrm{S} 1 \cdots \mathrm{Cg} 2 \cdots \mathrm{S} 2$ (first and second molecule from left to right) and $\mathrm{Cg} 2 \cdots \mathrm{S} 2 \cdots \mathrm{Cg} 1 \cdots \mathrm{S} 1$ (second and third molecule from left to right). The upper structural drawing is an example of an odd-member (i.c. BTBT-C5OH), whereas the lower structural drawing is an example of an even member (i.c. BTBT-C6OH). The lower and the upper vales for the even members are dependent of each other because of space group symmetry, whereas the values for the odd members are symmetry-independent. The markers are blue for the odd members and red for the even members.

The most remarkable odd-even effect in BTBT-CnOH is the rotation of one half of the BTBT cores of all even members by $180^{\circ}$ around their long axis in an inverted herringbone organization with only one type of $S \cdots S$ contact (edge-to-edge $S \cdots S$ contacts with $S \cdots S$ 


\section{WILEY-VCH}

distance in the order of $3.55 \AA$ ). This has already been discussed for the structure of BTBT-

$\mathbf{C 6 O H},{ }^{[16]}$ but can now be generalized as an odd-even effect. Figure 6 shows the herringbone patterns of all BTBT-CnOH members where the sulfur atoms which are in the background are orange colored and those in the foreground yellow.

Edge-to-edge S $\cdots \mathrm{S}$ contacts are present in the structures of both the odd and the even members with distances in the 3.46-3.62 A range, but the face-to-edge S $\cdots$ S contacts are absent for the even members. This visual observation can be quantified by calculating the torsion angles $\mathrm{Cg} 1 \cdots \mathrm{S} 1 \cdots \mathrm{Cg} 2 \cdots \mathrm{S} 2$ where $\mathrm{Cg} n$ refers to the center of gravity of the two carbon atoms opposite to the sulfur atom in a thiophene unit and the numbers $n$ designate two different thiophene units stacked in herringbone mode. This is shown in Figure $7 \mathrm{~b}$ where a very clear alternation is seen between odd and even members of the BTBT-CnOH series. The absence of the face-to-edge $S \cdots S$ contacts in the structures of the even BTBT-CnOH suggests that the mobility in these structures has a one-dimensional rather than a two-dimensional character as for the odd members and all members of the BTBT-Cn series.

The $180^{\circ}$ rotation of one half of the BTBT cores for the even members of BTBT-CnOH can be related to different hydrogen-bond networks for the odd and even members which in turn could be the result of the less compact space between adjacent molecular layers for the odd members compared to that of the even members. The analysis of the hydrogen-bond networks is a bit delicate, since the hydrogen bond networks are for all members, except for $n=5$, disordered. Figure 8 compares the hydrogen-bond networks of BTBT-C5OH and BTBT$\mathbf{C 6 O H}$, where in the latter case one of the two possible networks have been chosen. In both cases the hydrogen bond network consists of infinite parallel $\mathrm{C}_{2}^{2}(4)$ chains. An important difference is that in the case of BTBT-C5OH the chains are composed of pairs of hydrogen bonded $\mathrm{OH}$ groups in the top layer $(t)$ connected to pairs in the bottom layer $(b)$, whereas in 


\section{WILEY-VCH}

BTBT-C6OH the hydrogen-bonded chain crosses the gap at each individual OH group. In other words, the chain in BTBT-C5OH can be designated as

$\cdots \mathrm{OH}(t) \cdots \mathrm{OH}(t) \cdots \mathrm{OH}(b) \cdots \mathrm{OH}(b) \cdots$, whereas the BTBT-C6OH chain is $\cdots \mathrm{OH}(t) \cdots \mathrm{OH}(b) \cdots \mathrm{OH}(t) \cdots \mathrm{OH}(b) \cdots$
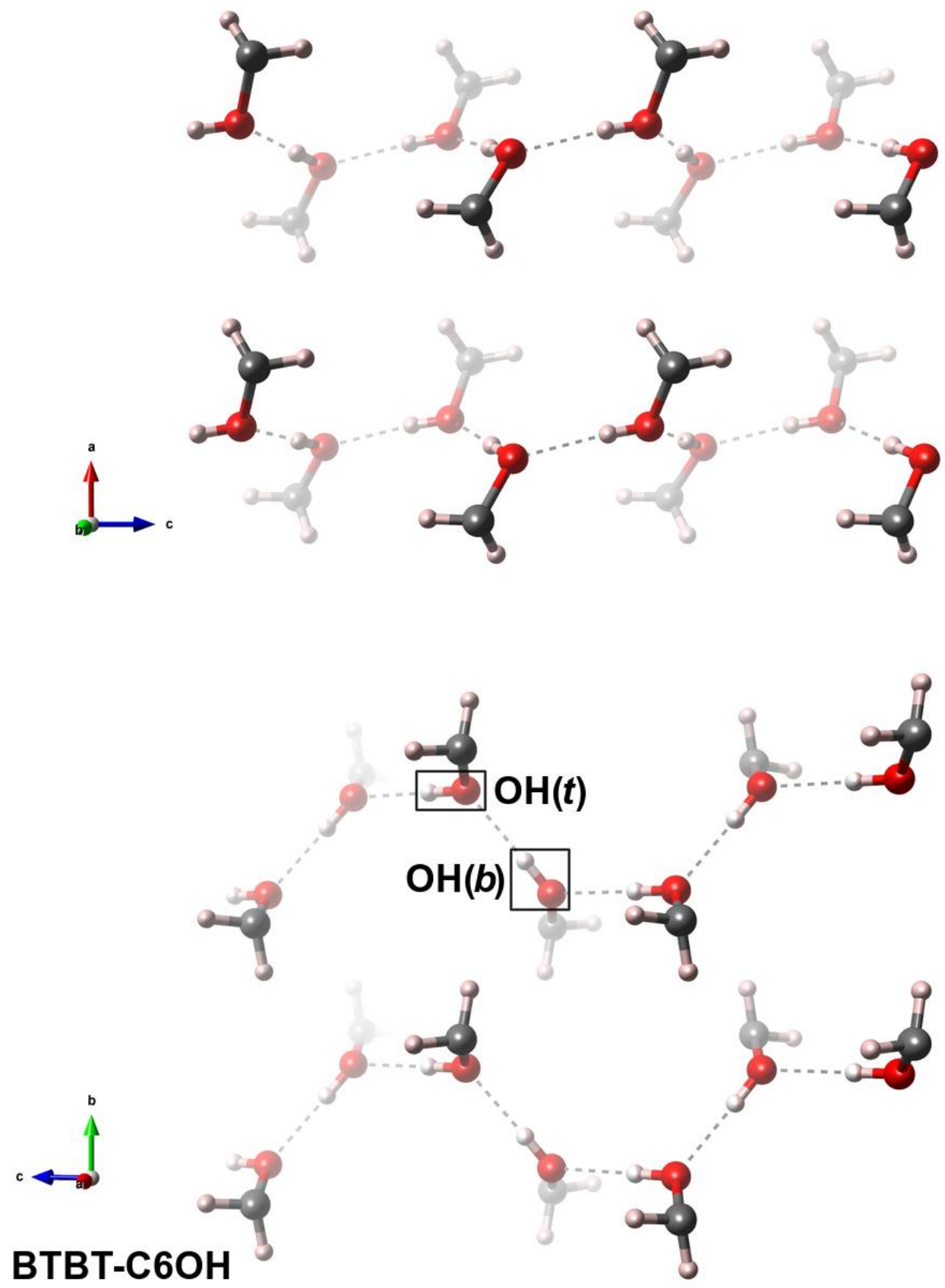


\section{WILEY-VCH}

Figure 8. Hydrogen-bond networks in the structures of BTBT-C5OH and BTBT-C6OH. The projection is in both cases parallel to the stacking direction of the molecular layers. For clarity only the terminal $\mathrm{CH} 2-\mathrm{OH}$ groups of adjacent molecular layers are shown. Depth cueing was used to fade away the $\mathrm{CH} 2-\mathrm{OH}$ groups in the bottom layer $(b)$ in the background from those in the top layer $(t)$ in the foreground. Two examples of $\mathrm{OH}$ groups in the top and bottom layer, respectively, have been indicated by small rectangles.

$\cdots \mathrm{OH}(t) \cdots \mathrm{OH}(t) \cdots \mathrm{OH}(b) \cdots \mathrm{OH}(b) \cdots$ chains are visible in BTBT-C5OH and $\cdots \mathrm{OH}(t) \cdots \mathrm{OH}(b) \cdots \mathrm{OH}(t) \cdots \mathrm{OH}(b) \cdots$ chains in BTBT-C6OH.

A second important difference between the odd and even members is the torsional freedom of the terminal $\mathrm{OH}$ groups. The even members have two identical tails in line with all members of the BTBT-Cn series, i.e. the torsion angles made up by the last four non-hydrogen atoms of the chain are all close to $\pm 180^{\circ}$ as the other internal torsion angles within the chain, i.e. the chains are practically unfolded and linear, whereas the two chains of the odd members are asymmetric with respect to the terminal torsion angles: one is close to $\pm 180^{\circ}$, whereas the opposite one is close to $\pm 60^{\circ}$. This is very clear in the completely ordered structure of BTBTC5OH, but can also be observed in the partially disordered structures of BTBT-C7OH and BTBT-C9OH. The rotation of one of the terminal alcohol groups makes it possible to set up intra-layer $\cdots \mathrm{OH}(t, b) \cdots \mathrm{OH}(t, b)$ hydrogen bond interactions.

The driving force for the supramolecular assembly is the set-up of the hydrogen-bonded network between the terminal hydroxyl groups, either between molecules within a layer or between layers, or both. The energetic 'choice' made by the even members is to maximize the interlayer interactions by rotating one out of two BTBT cores so that two pairs of hydroxyl groups come in a favorable position for a hydrogen-bond interaction crossing the interlayer gap. The odd members rotate one out of two terminal $\mathrm{OH}$ groups in order to establish an intralayer hydrogen-bond interaction, leaving one possibility for an interlayer interaction. The energetic difference between the two possibilities is small ( $0.6 \mathrm{eV}$ for 200 atoms) as was found from periodic DFT calculation by flipping one BTBT core from a classical to an inverted herringbone structure. ${ }^{[33]}$ 


\section{WILEY-VCH}

Having established the presence of a remarkable odd-even effect in the relative orientation of the BTBT cores, we turned our attention to the optoelectronic properties of BTBT-CnOH.

Figure 9a gives the normalized UV-vis absorbance spectra of the $n=5-10$ members, determined on thin films evaporated on glass substrates and the position of the absorbance maximum as a function of the number of methylene units $n$. All maxima are red-shifted with the increase of the alkyl chain length (Figure 9b). The positions of the absorbance maxima of the even members appear to modulate on those of the odd members which display a linear behavior. Two different structural effects are in fact superimposed, since the increasing length of the alkyl chains alone often results in a continuous red-shift of the maxima (although this is not always the case, e.g. for $\mathbf{B T T T}^{[13]}$ ), whereas the $180^{\circ}$ rotation of the BTBT cores between odd and even members alone should result in an alternating red-blue shift of the maxima.
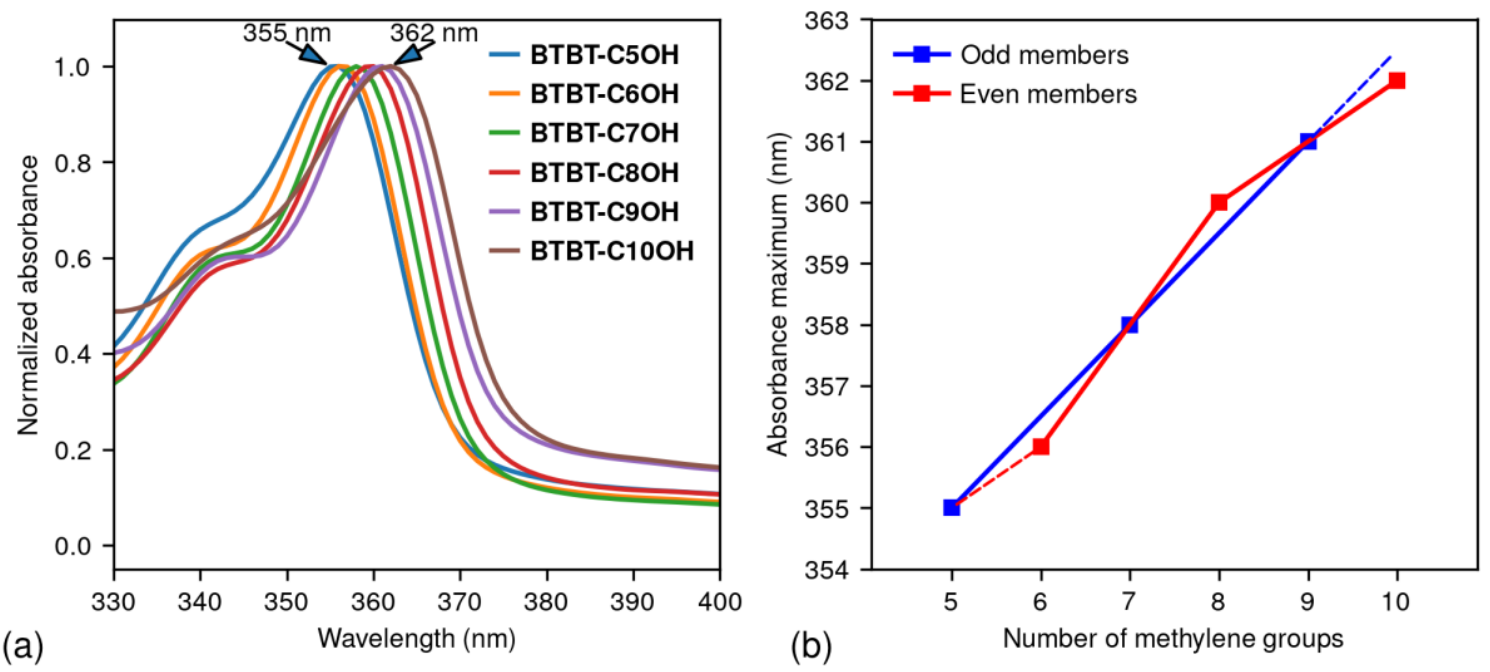

Figure 9. (a) UV-vis absorbance spectra realized on thin films of BTBT-CnOH evaporated on glass substrates. b) evolution of the absorbance maxima as function of the number of $\mathrm{CH}_{2}$ groups in BTBT-CnOH.

The charge carrier mobilities were investigated in field-effect transistors with a bottom gate/bottom contact (BG/BC) geometry. Since all BTBT-CnOH structures have the same lamellar organization and more importantly the same orientation with respect to the $\mathrm{Si} / \mathrm{SiO}_{2}$ substrates, the mobilities of BTBT-CnOH based OFETs could be compared. Figure 10 gives 


\section{WILEY-VCH}

the mobilities $\mu_{\mathrm{OFET}}$ measured on more than 8 devices for each member and Table 3 . FET characteristics of BTBT-CnOH devices fabricated on $\mathrm{Si} / \mathrm{SiO}_{2}$ gives the FET characteristics of BTBT-CnOH devices fabricated on $\mathrm{Si} / \mathrm{SiO}_{2}$, i.e. the average values of $\mu_{\mathrm{OFET}}, I_{\mathrm{on}} / I_{\mathrm{off}}$, and $V_{\text {th }}$ for each $n$. Figs. S60-S65 give the transfer curves in the linear and saturated regimes as well as the output curves for the BTBT-CnOH FET-devices. Figure 10 shows that the mobility sharply increases with the length of the alkyl chains, in contrast with the mobilities of BTBT$\mathrm{Cn}$ where the average increase is more moderate or nearly absent (Figure 2b). The two different superimposed structural effects (chains length and $180^{\circ}$ rotation) evidenced on the UV-vis absorbance spectra (Figure 9) also control the mobilities of BTBT-CnOH. According to the "Fastener Molecular" effect ${ }^{[34]}$, (i.e. an increase of the transfer integrals between the $\pi$-conjugated cores as the length of the alkyl chains increases), mobilities of both series (odd and even) should increase. ${ }^{[19]}$ However, mobilities of the complete BTBT-CnOH series do not increase linearly with the chain length, but show instead a clear odd-even effect, the mobilities of the odd members being in all cases higher than their even neighbors. The most striking difference is observed between the average mobility of BTBT-C8OH being at $0.12 \mathrm{~cm}^{2} \mathrm{~V}^{-1} \mathrm{~s}^{-1}$ whereas the mobility of BTBT-C9OH is nearly one order of magnitude higher at $1.04 \mathrm{~cm}^{2} \mathrm{~V}^{-1} \mathrm{~s}^{-1}$. This odd-even effect is certainly the result of the two types of herringbones (classical and inverted) found in the two different series (Fig. 6). While the classical herringbone exhibited by the odd series results in a two-dimensional S $\cdots S$ interaction network and as a consequence higher mobilities, the inverted herringbone found in the even series results in mono-dimensional network and consequently lower mobilities.

It is important to note that the devices based on BTBT-CnOH were highly reliable and reproducible even if the molecules were directly evaporated on the UVO-treated $\mathrm{SiO}_{2}$ dielectric substrate without the use of any passivation layer. Hydroxy - $\mathrm{SiO} 2$ interactions might direct and stabilize the BTBT-CnOH layers on the substrate. 


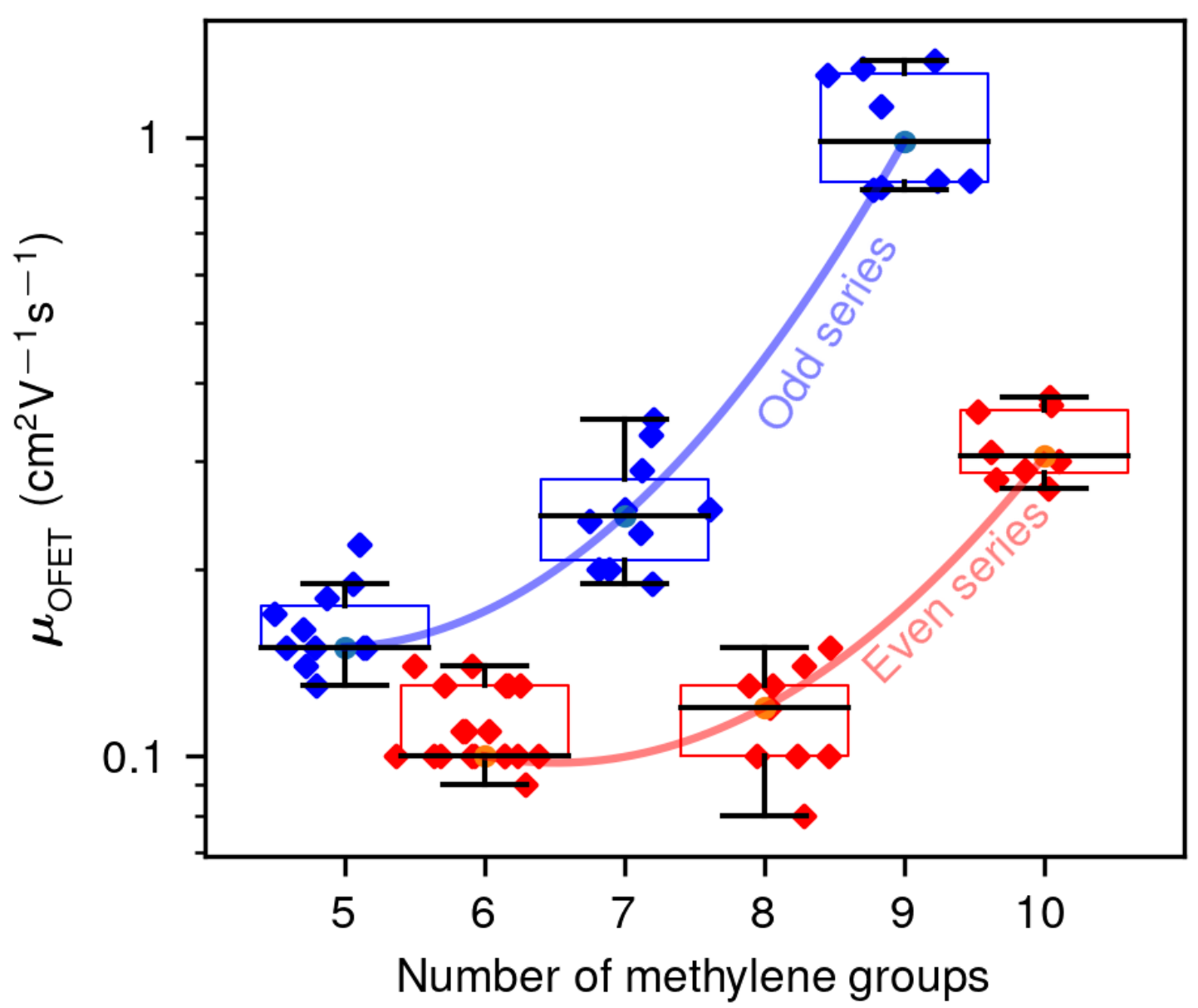

Figure 10. OFET mobilities obtained for the BTBT-CnOH series. The height of the boxes extends from the lower to the upper quartile values of the measured values for each $n$. The orange horizontal lines in the middle of each box give the median values for each $n$. The whiskers give the minimum and maximum values in each series of measurements, whereas the points outside the whiskers are fliers. Some horizontal jitter has been added to the data points for each $n$ in order to separate them.

Table 3. FET characteristics of BTBT-CnOH devices fabricated on $\mathrm{Si} / \mathrm{SiO}_{2}$

\begin{tabular}{|l|l|l|l|}
\hline BTBT-CnOH & $\begin{array}{l}\mu_{\mathrm{OFET}} \\
\left(\mathrm{cm}^{2} \mathrm{~V}^{-1} \mathrm{~s}^{-1}\right)\end{array}$ & $I_{\mathrm{on}} / I_{\mathrm{off}}$ & $\begin{array}{l}V_{\mathrm{th}} \\
(\mathrm{V})\end{array}$ \\
\hline BTBT-C5OH & $0.16(2)$ & $10^{7}$ & +3 \\
BTBT-C6OH & $0.11(1)$ & $10^{8}$ & 0 \\
BTBT-C7OH & $0.25(5)$ & $10^{7}$ & +2 \\
\hline
\end{tabular}




\begin{tabular}{|l|l|l|l|} 
BТBT-C9OH & $1.0(2)$ & $10^{9}$ & +5 \\
BTBT-C10OH & $0.32(4)$ & $10^{8}$ & -5 \\
\hline
\end{tabular}

Notes: Standard deviations estimated from measurements on different devices are given in parentheses on the scale of the least significant digits of the numerical value.

\section{Conclusions}

We have designed a new family of compounds consisting of BTBT cores appended with variable-length alkyl chains and terminated by a structure directing hydroxyl group. The oddeven structural effect which is inherently present in layered alkyl chain containing compounds is dramatically amplified through the use of a structure-directing terminal hydroxyl group which causes the BTBT-cores to have alternating relative orientations depending on whether $n$ is odd or even. An unprecedented directed $180^{\circ}$ rotation of the optoelectronically active moiety as a function of the odd or even number of methylene spacers results in a pronounced odd-even effect on the semiconducting properties of the BTBT-CnOH family of compounds. For the first time, the odd-even effect controls the performances of devices. The alternating odd-even intra-layer assembly directly affects the transport properties in Organic Field Effect Transistors. The odd series shows significantly higher mobilities than the even series, because the conduction in the former series is two-dimensional rather than one-dimensional as for the even members. The use of structure directing terminal groups as an odd-even tool could be useful for other organic semiconductors to turn one-dimensional transport into twodimensional transport.

More insight in how the packing influences the mobilities can be obtained from calculations of intermolecular interaction energies and transfer integrals; this will be the topic of a forthcoming study.

\section{Experimental section}




\section{WILEY-VCH}

\subsection{Synthesis and NMR characterization}

All chemicals and solvents were of reagent grade unless otherwise indicated.

Dichloromethane (DCM) and N,N-dimethylformamide (DMF) were dried over $4 \AA$ molecular sieves and acetic acid over $3 \AA$ molecular sieves. Tetrahydrofuran (THF) was distilled under nitrogen over sodium benzophenone ketyl. BTBT and BTBT-C6OH were synthesized as described in our previous work ${ }^{[16]}$. All reactions were performed under nitrogen. Detailed descriptions of the syntheses can be found in the Supplementary Material, Section I. ${ }^{1} \mathrm{H}$ and ${ }^{13} \mathrm{C}$ Nuclear Magnetic Resonance (NMR) spectra were recorded on a Bruker AC $400 \mathrm{MHz}$ spectrometer (Figs S1-S41).

\subsection{X-ray data collection, structure solution and refinement.}

Single-crystal X-ray diffraction data for BTBT-C5OH were collected at the European Synchrotron Radiation Facility ID29 beamline $(\lambda=0.7290 \AA$ A $)$ in Grenoble, France; for BTBTC6OH and BTBT-C10OH on a Rigaku-Oxford Diffraction Gemini-S diffractometer $(\lambda$ $=0.7107 \AA$ ); for BTBT-C7OH on a Bruker Venture diffractometer $(\lambda=1.5418 \AA$ ), for BTBTC6, BTBT-C7, BTBT-C9, BTBT-C8OH, and BTBT-C9OH at the Elettra synchrotron XRD1 and XRD2 beamlines $(\lambda=0.70000 \AA)$, Trieste, Italy. Since the functional devices operate at room temperature, we collected most of the data at that temperature. For some of the structures the data collected at room temperature did not result in acceptable chemical structures and therefore data were collected at lower temperatures. For example, the structure of BTBT-C7OH was refined at room temperature to $R_{F}(I>2 \sigma(I))=0.261$ whereas at $173 \mathrm{~K}$ it converged to $R_{F}(I>2 \sigma(I))=0.088$. The general features of the two structures appeared to be very similar. More details about the experimental conditions and the structural refinements can be found in Table S2. CCDC 2059368-2059375 contains the supplementary crystallographic data in cif-format for the new structures reported in this paper. These data 


\section{WILEY-VCH}

can be obtained free of charge from The Cambridge Crystallographic Data Centre via www.ccdc.cam.ac.uk/structures

The thin film diffraction experiments were carried out on a PanAlytical X'pert-Pro diffractometer with $\mathrm{Ni}$-filtered $\mathrm{Cu}-K \alpha$ radiation $\lambda=1.5418 \AA$ ) in symmetric Bragg-Brentano configuration, assessing thus the $d$-spacings parallel to the surface.

\subsection{Organic Field Effect Transistors characterization}

OFETs were fabricated on Gen.4 chips from Fraunhofer IPMS (Dresden, Germany) with a bottom-gate and bottom-contact structure. The chips consist of a highly conductive n-doped Si wafer with a $230 \mathrm{~nm}$ thick thermally grown oxide. $\mathrm{SiO}_{2}$ exhibits a capacity of $14.63 \mathrm{nF} . \mathrm{cm}$

${ }^{2}$. On top of the $\mathrm{SiO}_{2}$ layer, a $10 \mathrm{~nm}$ thick ITO layer and a $30 \mathrm{~nm}$ thick Au layer were patterned to create source and drain contact electrodes in an interdigitated finger structure. Transistors have a channel length of $20 \mu \mathrm{m}$, a channel width of $10 \mathrm{~mm}$ and a common gate contact located on the bottom of the chip. Initially, substrates were cleaned in ultrasonic baths of acetone, isopropanol and DI water for 5 minutes each at room temperature and treated with ozone plasma for an additional 15 min and quickly introduced in a glove box. Finally, a 20 nm thick film of BTBT-CnOH was evaporated at $1 \AA /$ min followed by $30 \mathrm{~nm}$ at $12 \AA / \mathrm{min}$, affording the operating device. Electrical characterizations of the fabricated OFETs (Fig. S59) were performed using a Keithley 4200 Semiconductor Characterization System in a glove box environment under nitrogen atmosphere. Field effect mobilities $\mu_{\text {OFET }}$ were estimated from the saturated regime using the method described by Newman et al. ${ }^{[35]}$

\section{Supporting Information}

Supporting Information is available from the Wiley Online Library or from the author. 
- Syntheses of BTBT-Cn

- Syntheses of BTBT-CnOH

- Thermal gravimetric analyses

- Differential scanning calorimetry

- X-ray data collection, structure solution and refinement

- Procedure for the fabrication of the organic field effect transistors

- Characteristics of the organic field effect transistors

\section{Acknowledgements}

The authors acknowledge the ESRF (European Synchrotron Radiation Facility, Grenoble, France) for the allocation of beam time on ID29 and are grateful to David Flot for collecting synchrotron X-ray data for BTBT-C5OH on this beamline. GHR gratefully acknowledges financial support of ANR grant Helios $N^{\circ}$ ANR-13-PRGE-0006 of the PROGELEC program.

Received: ((will be filled in by the editorial staff))

Revised: ((will be filled in by the editorial staff)) Published online: ((will be filled in by the editorial staff))

\section{References}

[1] A. Baeyer, Berichte der deutschen chemischen Gesellschaft 1877, 10, 1286; W. E. Garner, F. C. Randall, J. Chem. Soc., Trans. 1924, 125, 881; C. W. Bunn, in Fibres from Synthetic Polymers, , (Ed: R. Hill), Elsevier, 1953; D. R. Holmes, C. W. Bunn, D. J. Smith, Journal of Polymer Science 1955, 17, 159; W. E. Garner, F. C. Madden, J. E. Rushbrooke, J. Chem. Soc. 1926, 129, 2491; P. Mukerjee, Kolloid-Zeitschrift \& Zeitschrift für Polymere 1970, 236, 76; S. Chandra, B. Bahadur, Journal de Chimie Physique 1973, 70, 605; J. W. Emsley, G. R. Luckhurst, G. N. Shilstone, Molecular Physics 1984, 53, 1023; A. D. Bond, New J. Chem. 2004, 28, 104; M. Qiu, K. M. Liew, The Journal of Physical Chemistry C 2012, 116, 11709; N. Nerngchamnong, L. Yuan, D.-C. Qi, J. Li, D. Thompson, C. A. Nijhuis, Nature Nanotechnology 2013, 8, 113; P. Yadav, A. Ballabh, New Journal of Chemistry 2015, 


\section{WILEY-VCH}

39, 721; J. A. Pradeilles, S. Zhong, M. Baglyas, G. Tarczay, C. P. Butts, E. L. Myers, V. K. Aggarwal, Nature Chemistry 2020, 12, 475.

[2] R. Boese, H.-C. Weiss, D. Bläser, Angewandte Chemie International Edition 1999, 38, 988.

[3] F. Tao, S. L. Bernasek, Chemical Reviews 2007, 107, 1408.

[4] L. Jiang, C. S. S. Sangeeth, C. A. Nijhuis, Journal of the American Chemical Society 2015, 137, 10659; M. Baghbanzadeh, F. C. Simeone, C. M. Bowers, K.-C. Liao, M. Thuo, M. Baghbanzadeh, M. S. Miller, T. B. Carmichael, G. M. Whitesides, Journal of the American Chemical Society 2014, 136, 16919; M. M. Thuo, W. F. Reus, C. A. Nijhuis, J. R. Barber, C. Kim, M. D. Schulz, G. M. Whitesides, Journal of the American Chemical Society 2011, 133, 2962.

[5] Y.-T. Tao, K.-Y. Wu, K.-H. Huang, T.-P. Perng, Organic Electronics 2011, 12, 602.

[6] Y. Yang, A. C. Jamison, D. Barriet, T. R. Lee, M. Ruths, Journal of Adhesion Science and Technology 2010, 24, 2511.

[7] Y. Feng, E. R. Dionne, V. Toader, G. Beaudoin, A. Badia, The Journal of Physical Chemistry C 2017, 121, 24626.

[8] J. T. E. Quinn, J. Zhu, X. Li, J. Wang, Y. Li, Journal of Materials Chemistry C 2017, 5, 8654; G. Gryn'ova, K.-H. Lin, C. Corminboeuf, Journal of the American Chemical Society 2018, 140, 16370; J. Borges-González, C. J. Kousseff, C. B. Nielsen, Journal of Materials Chemistry C 2019, 7, 1111; H. Bronstein, C. B. Nielsen, B. C. Schroeder, I. McCulloch, Nature Reviews Chemistry 2020, 4, 66.

[9] H. Ebata, T. Izawa, E. Miyazaki, K. Takimiya, M. Ikeda, H. Kuwabara, T. Yui, Journal of the American Chemical Society 2007, 129, 15732.

[10] T. Izawa, E. Miyazaki, K. Takimiya, Advanced Materials 2008, 20, 3388.

[11] G. Schweicher, V. Lemaur, C. Niebel, C. Ruzié, Y. Diao, O. Goto, W. Y. Lee, Y.

Kim, J. B. Arlin, J. Karpinska, A. R. Kennedy, S. R. Parkin, Y. Olivier, S. C. B. Mannsfeld, J. Cornil, Y. H. Geerts, Z. Bao, Advanced Materials 2015, 27, 3066.

[12] H. Chen, Y. Guo, G. Yu, Y. Zhao, J. Zhang, D. Gao, H. Liu, Y. Liu, Advanced Materials 2012, 24, 4618; T. Lei, J.-Y. Wang, J. Pei, Chemistry of Materials 2013, 26, 594; S. Chen, B. Sun, W. Hong, H. Aziz, Y. Meng, Y. Li, J. Mater. Chem. C 2014, 2, 2183; Z. Ma, H. Geng, D. Wang, Z. Shuai, Journal of Materials Chemistry C 2016, 4, 4546; B. C. Schroeder, T. Kurosawa, T. Fu, Y.-C. Chiu, J. Mun, G.-J. N. Wang, X. Gu, L. Shaw, J. W. E. Kneller, T. Kreouzis, M. F. Toney, Z. Bao, Advanced Functional Materials 2017, 27, 1701973; M. Moral, A. Navarro, A. Garzón-Ruiz, E. M. García-Frutos, The Journal of Physical Chemistry C 2018, 123, 153.

[13] E. K. Burnett, Q. Ai, B. P. Cherniawski, S. R. Parkin, C. Risko, A. L. Briseno, Chemistry of Materials 2019, 31, 6900.

[14] T. Uemura, Y. Hirose, M. Uno, K. Takimiya, J. Takeya, Applied Physics Express 2009, 2, 111501; H. Minemawari, T. Yamada, H. Matsui, J. y. Tsutsumi, S. Haas, R. Chiba, R. Kumai, T. Hasegawa, Nature 2011, 475, 364; K. Nakayama, Y. Hirose, J. Soeda, M. Yoshizumi, T. Uemura, M. Uno, W. Li, M. J. Kang, M. Yamagishi, Y. Okada, E. Miyazaki, Y. Nakazawa, A. Nakao, K. Takimiya, J. Takeya, Advanced Materials 2011, 23, 1626; K. Takimiya, T. Yamamoto, H. Ebata, T. Izawa, Thin Solid Films 2014, 554, 13; Y. Yuan, G. Giri, A. L. Ayzner, A. P. Zoombelt, S. C. B. Mannsfeld, J. Chen, D. Nordlund, M. F. Toney, J. Huang, Z. Bao, Nature Communications 2014, 5; K. Takimiya, I. Osaka, T. Mori, M. Nakano, Accounts of Chemical Research 2014, 47, 1493; O. V. Borshchev, A. S. Sizov, E. V. Agina, A. A. Bessonov, S. A. Ponomarenko, Chemical Communications 2017, 53, 885. [15] D. G. Dumitrescu, G. H. Roche, J. J. E. Moreau, O. J. Dautel, A. van der Lee, Acta Crystallographica Section B Structural Science, Crystal Engineering and Materials 2020, 76, 661. 


\section{WILEY-VCH}

[16] G. H. Roche, Y.-T. Tsai, S. Clevers, D. Thuau, F. Castet, Y. H. Geerts, J. J. E. Moreau, G. Wantz, O. J. Dautel, Journal of Materials Chemistry C 2016, 4, 6742.

[17] A. van der Lee, G. H. Roche, G. Wantz, J. J. E. Moreau, O. J. Dautel, J.-S. Filhol, Chemical science 2018, 9, 3948.

[18] M. D. Curtis, J. Cao, J. W. Kampf, Journal of the American Chemical Society 2004, 126, 4318; K. Takimiya, T. Ogaki, C. Wang, K. Kawabata, Chemistry - An Asian Journal 2020, 15, 915 .

[19] H. Minemawari, M. Tanaka, S. Tsuzuki, S. Inoue, T. Yamada, R. Kumai, Y. Shimoi, T. Hasegawa, Chemistry of Materials 2017, 29, 1245.

[20] International Tables for Crystallography, International Union of Crystallography, 2016.

[21] R. B. Helmholdt, R. Peschar, H. Schenk, Acta Crystallographica Section B Structural Science 2001, 58, 134.

[22] D. P. van Heerden, L. J. Barbour, Chemical Society Reviews 2021.

[23] A. I. Kitajgorodskii, Molecular Crystals and Molecules, Academic Press, New-York 1973.

[24] C. F. Macrae, I. Sovago, S. J. Cottrell, P. T. A. Galek, P. McCabe, E. Pidcock, M. Platings, G. P. Shields, J. S. Stevens, M. Towler, P. A. Wood, Journal of applied crystallography 2020, 53, 226.

[25] C. Sutton, M. S. Marshall, C. D. Sherrill, C. Risko, J.-L. Brédas, Journal of the American Chemical Society 2015, 137, 8775.

[26] A. Y. Amin, A. Khassanov, K. Reuter, T. Meyer-Friedrichsen, M. Halik, Journal of the American Chemical Society 2012, 134, 16548.

[27] M. M. Walczak, C. Chung, S. M. Stole, C. A. Widrig, M. D. Porter, Journal of the American Chemical Society 1991, 113, 2370; S.-C. Chang, I. Chao, Y.-T. Tao, Journal of the American Chemical Society 1994, 116, 6792.

[28] M. D. Porter, T. B. Bright, D. L. Allara, C. E. D. Chidsey, Journal of the American Chemical Society 1987, 109, 3559; A. Ulman, Chemical Reviews 1996, 96, 1533; S. Lee, A. Puck, M. Graupe, R. Colorado, Y.-S. Shon, T. R. Lee, S. S. Perry, Langmuir 2001, 17, 7364. [29] F. Tao, S. L. Bernasek, The Journal of Physical Chemistry B 2005, 109, 6233; M. Hibino, A. Sumi, H. Tsuchiya, I. Hatta, The Journal of Physical Chemistry B 1998, 102, 4544.

[30] K. Morishige, T. Kato, The Journal of Chemical Physics 1999, 111, 7095.

[31] G. Mukherjee, K. Biradha, Crystal Growth \& Design 2011, 11, 924; G. Mukherjee, K. Biradha, Journal of Chemical Sciences 2014, 126, 1285; V. Kocasoy, B. Dedeoglu, O. DemirOrdu, V. Aviyente, RSC Advances 2018, 8, 35195.

[32] A. Paul, M. Kubicki, Journal of Molecular Structure 2009, 938, 238; A. Paul, M. Kubicki, Journal of Chemical Crystallography 2011, 41, 703; G. Dutkiewicz, E. Dutkiewicz, M. Kubicki, Structural Chemistry 2014, 26, 247.

[33] G. H. Roche , D. Flot , J. J. E. Moreau , O. J. Dautel , J.-S. Filhol , Van der Lee, A. Submitted 2021.

[34] H. Inokuchi, G. Saito, P. Wu, K. Seki, T. B. Tang, T. Mori, K. Imaeda, T. Enoki, Y. Higuchi, K. Inaka, N. Yasuoka, Chemistry Letters 1986, 1263; H. Inokuchi, K. Imaeda, T. Enoki, T. Mori, Y. Maruyama, G. Saito, N. Okada, H. Yamochi, K. Seki, Y. Higuchi, N. Yasuoka, Nature 1987, 329, 39.

[35] C. Newman, C. Frisbie, D. da Silva, J. Bredas, P. Ewbank, K. Mann, Chemistry of Materials 2004, 16, 4436.

Table of Contents entry : 


\section{WILEY-VCH}

An unprecedented odd-even alternation in the relative orientation of $\pi$-conjugated cores in the structures of organic semiconductors as a function of the number of methylene spacers in the side alkyl chains changes the dimensionality of carrier transport from one- to two-dimensional.

Structural Odd-Even Effect Impacting the Dimensionality of Transport in BTBT-CnOH Organic Field Effect Transistors

ToC figure ((Please choose one size: $55 \mathrm{~mm}$ broad $\times 50 \mathrm{~mm}$ high or $110 \mathrm{~mm}$ broad $\times 20 \mathrm{~mm}$ high. Please do not use any other dimensions))

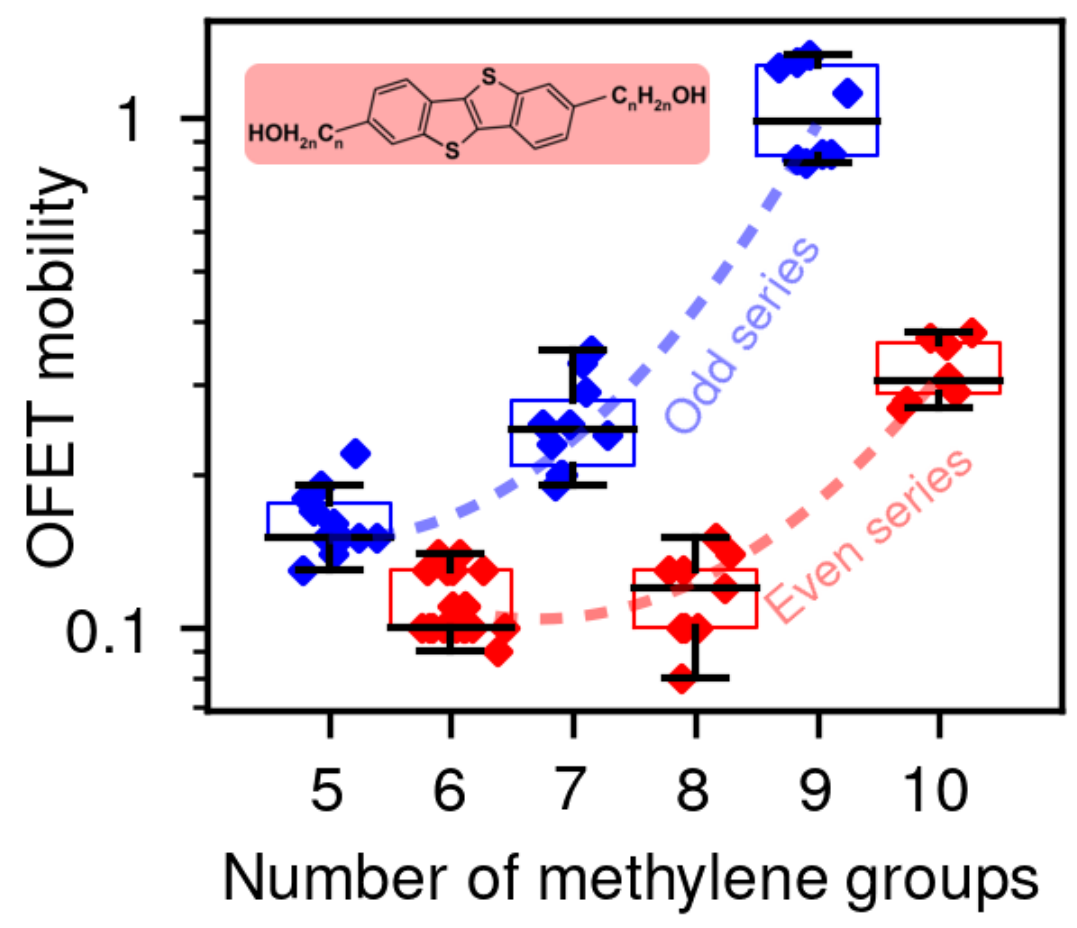




\section{WILEY-VCH}

Supporting Information

Structural Odd-Even Effect Impacting the Dimensionality of Transport in BTBT-CnOH Organic Field Effect Transistors

Author(s), and Corresponding Author(s)*((write out full first and last names)) 